\title{
Comparative physiological and biochemial effects of CuO NPs and bulk CuO phytotoxicity onto the maize (Zea mays) seedlings
}

\author{
El-Shazoly R.M. ${ }^{1}$ and Amro A. ${ }^{2}$ \\ ${ }^{1}$ Botany and Microbiology Department, Faculty of Science, New Valley University, 72511, Al-Kharja, New Valley, Egypt \\ ${ }^{2}$ Botany and Microbiology Department, Faculty of Science, Assiut University, Assiut 71516, Egypt \\ Received: 15/05/2018, Accepted: 19/11/2018, Available online: 21/11/2018 \\ https://doi.org/10.30955/gnj.002768
}

\begin{abstract}
Plant nanotoxicology is an emerging and less-explored area of research for the plant stress biologists. The present study assesses the toxicity of bulk-CuO and nano-CuO (size $<50 \mathrm{~nm}$ and surface area $=29 \mathrm{~m}^{2} \mathrm{~g}^{-1}$ ) on maize (Zea mays cv. hybrids triple white $\mathrm{Hi}$ Tech.) seedlings. Five successive levels of stress $(25,50,100,150$ and $200 \mathrm{mg} \mathrm{L}^{-1}$ ) suspensions of $\mathrm{CuO}$ and CuONP were imposed and seedling growth performance was studied along control at 8 days of experiment. Modulation of enzymatic antioxidants (SOD, CAT, APX and POD) and non-enzymatic antioxidants (total phenolics and total flavonoids) activities under both-CuO stresses were investigated in detail to get an overview of their-stress response of maize. Seed germination was completely stopped under 150 and $200 \mathrm{mg} \mathrm{L}^{-1}$ CuONP. Generally, CuONP stress was most violent and $100 \mathrm{mg} \mathrm{L}^{-1}$ CuONP showed the same or more drastic effect in 150 and $200 \mathrm{Cu}$ bulk. Photosynthetic pigments, shoot and root lengths reduced under both stresses by about $50 \%$ of control. Soluble proteins and total antioxidants increased gradually till it reached about $200 \%$ of control at $150 \mathrm{mg} \mathrm{L}^{-1} \mathrm{CuO}$ and about $200-300 \%$ at $100 \mathrm{mg} \mathrm{L}^{-1} \mathrm{CuONP}$. In addition, increased reducing power activity coupled with both stresses which reached to $400 \%$ of control at $150 \mathrm{mg} \mathrm{L}^{-1} \mathrm{CuO}$ and $100 \mathrm{mg} \mathrm{L}^{-1}$ CuONP. Moreover, superoxide dismutase increased by $800 \%$ of control at 100 $\mathrm{mg} \mathrm{L}^{-1}$ CuONP.
\end{abstract}

Keywords: Nanotoxicology, $\mathrm{CuO}$, oxide, reducing power, $\mathrm{H}_{2} \mathrm{O}_{2}$ scavenging\%, metal chelating\%, multivariate analysis.

\section{Introduction}

Advances in nanotechnology include the incorporation of many metallic nanoparticles (NPs) into diverse industrial, household and medical products (Lee et al., 2008; Navarro et al., 2008). Although, many of them are useful, some are toxic to micro and macro-flora, including plants and our food-crops.Improper handling and disposal of NPcontaining wastes could result in environmental contamination. Uncontrolled release of metal oxide nanoparticles (NPs) into the environment due to human activities has become a serious threat to the ecological system.

The development of nanotechnology in physiology and biochemistry has expanded the application area of nano-materials in different fields due to their unique characters such as large surface areatovolume ratio, ability to engineer electron exchange and highly surface reactive capability (Scrinis and Lyons, 2007).

In recent years, many scientists have studied the effects of these materials on seed germination and plant growth with the aim to promote its use for agricultural applications. Most of these studies are focused on the potential phyto-toxicity of nanoparticles in higher plants and both positive and subsequently negative or inconsequential effects were represented. NPs have the potential for harmful effects on plants and their associated soil microflora (Dimkpa et al., 2011; Lin and Xing, 2008; Nair et al., 2010). At sub-lethal concentrations, NPs variably modify the production of secondary metabolites from bacterial that involved in plant growth and production (Dimkpa et al., 2012) andmay pose a route for contamination of the food chain (Hatami and Ghorbanpour, 2014).

Some nano-materials are toxic to flora and fauna as they are used to inhibit their growth to prevent further multiplication (Siddiqi and Husen, 2016a). The toxicity response depends on the concentration, particle shape and size of the se nano-materials (Siddiqi and Husen, 2016b). Toxicity of NPs depends on many factors such as their conformation, surface characteristics, such as the presence of coatings, and their state of aggregation (Barrena et al., 2009). The impact of nanoparticles on higher plants appears to depend on the species and age of the plants, the experimental conditions such as temperature, the duration and method of exposure. Bio-uptake and accumulation of nano-materials in plants may increase shoot height and decrease root length (Atha et al., 2012), and they recorded to be reach to the shoots (Lee et al., 2008). The pathway of water and nutrienttransport has one mechanism proposed to account for how the NPs cause their damage to the plant (Lin and 
Xing, 2008). In addition, these metallic NPs may release soluble metals (Lee et al., 2008; Lin and Xing, 2008) that are taken up by the plant.

Growth of food plants such as lettuce, cucumber, bean, rye, corn, and zucchini is impaired, depending on the concentration of $\mathrm{Cu}, \mathrm{Ag}, \mathrm{CuO}, \mathrm{Zn}, \mathrm{TiO}_{2}$, and $\mathrm{ZnO}$ NPs (Atha et al., 2012; Barrena et al., 2009; Du et al., 2011). It was shown that 40 and $60 \mathrm{mg} \mathrm{L}^{-1}$ NanoSilver-treated Pelargomium zonale encourage an efficient cellular electron exchange mechanism, which slows down electron leakage and consequently reduces the ROS production and malonaldehyde (MDA) content (Lu et al., 2002). It was reported that activity of specific antioxidant enzymes was induced in Brassica juncea seedlings treated with silver nanoparticles (Priyadarshini et al., 2012). For this instance, $10 \mathrm{mgL}^{-1}$ AgNPs was found to inhibit seed germination in Hordeum vulgareand reduced shoot length in flax and barley (El-Temsah and Joner, 2012).

Exposure to ions such as $\mathrm{Al}, \mathrm{Cd}, \mathrm{Fe}, \mathrm{Mn}, \mathrm{Cu}, \mathrm{Ni}, \mathrm{Zn}$, and $\mathrm{U}$ results in many symptoms including loss of chlorophyll pigmentation, increased lipid membrane peroxidation, altered ferric iron metabolism, modification in the levels of plant growth regulators and activities of stress-inducible enzymes (Dimkpa et al., 2008).

Many studies found that physiological indexes were positively affected by nanoparticle treatments during thermal treatments (Mohammadi et al., 2013). Moreover, it was shown that Titanium nanoparticles $\left(\mathrm{TiO}_{2}\right)$ not onlyreducedoxidative damage but also alleviated membrane damage indexes (electrolyte leakage) under cold stress treatment in chickpea genotypes. In spinach, $\mathrm{TiO}_{2}$ NPs increase RUBISCO activase that enhance photosynthesis and plant growth (Gao et al., 2008).

Keller and Lazareva (2013) have reported that about 5500-3000 tons of TiO2NPs are produced every year and more than $50 \%$ of which is used in personal care products followed by 550 tons/year for ZnONP and Ag NPs is about 55 tons (Piccinno et al., 2012). Copper-based nanoparticles (NPs) are profusely used due to their optical, thermal, electrical, antibacterial, and catalytic applications (Peralta-Videa et al., 2016). Its global production in 2010 was estimated in 200 tons, 36 of which ended up in soil and 11 in bodies of water (Keller et al., 2013). Very recently, nanoparticulate forms of copper are starting to be used in agriculture for specific purposes. For instance, it has been used as alternative to bulk-Cu products to combat fungal diseases (Servin et al., 2015). The production of $\mathrm{Cu}$-based nano forms is predicted to reach to unanticipated levels. However, little is known about their physiological and biochemical effects in many agricultural crops (Du et al., 2017). From previous publications, it is known that nano-CuO, at different concentrations, alters plant growth and development by increasing the reactive oxygen species (ROS) production and unbalancing homeostasis of essential elements (Du et al., 2017).

Shaw et al. (2014) have shown that CuO nanoparticles reduced shoot and root growth of Hordeum vulgare seedlings. They have also reported that the CuO NPs induced the release of ROS, membrane damage and overall enzymatic activity not enough to cope with stress at 20-day exposure.It has been proposed that $\mathrm{CuO}$ nanoparticles would have been translocated via the vascular tissues and subsequently dissolved to produce $\mathrm{Cu}$ ions which resulted in deposition of lignin. Translocation of $\mathrm{CuO}$ nanoparticles is apparent but the production of $\mathrm{Cu}$ ions by dissolution is impossible because generation of $\mathrm{Cu}$ ions from copper nanoparticles is a redox process which requires a reducing agent such as hydrogen, phenol, protein or an acid (Siddiqi and Husen, 2017). The plants grown in presence of nanoparticles may absorb and translocate them in different tissues. It has been shown that $\mathrm{CuO}$ nanoparticles were reduced to $\mathrm{Cu}_{2} \mathrm{O}$ and $\mathrm{Cu}_{2} \mathrm{~S}$ in maize plants (Wang et al., 2012).

Duckweed exposed to CuO nanoparticles showed inhibition of photosynthetic activity due to the $\mathrm{Cu}^{2+}$ ions released from it (Perreault et al., 2014). Carotenoids remained unchanged and chlorophyll reduction began at $100 \mathrm{mgL}^{-1} \mathrm{CuO}$ nanoparticles in mung beans (Nair et al., 2014). Chlorophyll started decreasing at $100 \mathrm{mg} \mathrm{L}^{-1}$ (Nair and Chung, 2014). In another study, CuO nanoparticles reduced carotenoids and chlorophylls in mustard (Nair and Chung, 2015).

Copper oxide NPs have been shown to induce DNA damage in plants (Atha et al., 2012). Growth inhibition in Raphanus sativus, Lolium perenne and Lolium rigidum under laboratory conditions has been reported. Germination of radish seeds in presence of $\mathrm{CuO}$ nanoparticles induces substantial accumulation of mutagenic DNA lesions. Radish and similar other plants produce oxygen-derived species $\left(\mathrm{O}_{2}^{-}, \mathrm{H}_{2} \mathrm{O}_{2}, \mathrm{OH} \cdot\right)$ during germination (Schopfer et al., 2001). $\mathrm{H}_{2} \mathrm{O}_{2}$ enhances seed germination but in presence of peroxidase or transition metal ions such as copper produce an excess of $\mathrm{OH}$ - via the Fenton reaction (Halliwell and Gutteridge, 2007). It is therefore suggested that copper ions produced from $\mathrm{CuO}$ NPs may catalyze the formation of this free radicle. CuO NPs inhibited the radish root growth to the extent of $79 \%$ which is relatively much larger than that observed for $\mathrm{Cu}_{2}{ }^{+}$ ions alone. The stunted growth has been observed mainly in the root/shoot (Fernandes and Henriques, 1991).

Plants are generally protected against this oxidative stresses by a wide range of radical scavenging systems such as antioxidative enzymes like peroxidase, ascorbate peroxidase superoxide dismutase and catalase as well as nonenzymatic compounds include antioxidants such as ascorbate, glutathione, $\alpha$-tocopherol and carotenoids (Azevedo Neto et al., 2008; Zimmermann and Zentgraf, 2005). These components minimize the oxidative damage during exposure to metal oxide nanoparticles (Zhao et al., 2012a). Therefore, controlling ROS levels would be clearly advantageous in improving the plant performance and longevity. Zea mays exposed to $\mathrm{CeO}_{2} \mathrm{NPs}$ (Zao et al., 2012b) did not show lipid peroxidation and any physiological changes, although activity of catalase, ascorbate and upregulation of heat shock proteins was observed. However, no elevation of lipid peroxidation in 
rice treated with this compound $\left(0-500 \mathrm{mg} \mathrm{L}^{-1}\right)$ was recorded but ion leakage was observed at higher doses (Rico et al., 2013).

The scientific information on potential harmful effects of NPs severely lags behind the development of nanotechnologies (Kahru and Ivask, 2013). The available nanotoxicity data are inconsistent because experimental approaches vary from article to article making it impossible to compare results (Schrurs and Lison, 2012). To overcome these problems, nanotoxicology community has recently started a discussion about the implementation of general guidelines for nano-toxicology research and establishment of common parameters that should be addressed in all nano-toxicological articles.

The present study was carried out to elucidate the potential effects of bulk (CuO) and nano-Copper (CuONP) particle application on photosynthetic pigments, seedling development, lipid peroxidation inhibition\%, LOX, protein content, defense antioxidants (enzymatic and nonenzymatic) activity and metal chelating\% in maize (Zea mays).

\section{Materials and methods}

\subsection{CuO nanoparticle and bulk particle suspension} preparation

CuO Nanoparticle (CuONP) tested for phytotoxicity was purchased from Sigma-Aldrich. Specific surface area, size, and purity of the compound were adopted (where available) from the manufacturer. CuO (size $<50 \mathrm{~nm}$, surface area $\left.=29 \mathrm{~m}^{2} \cdot \mathrm{g}^{-1}\right)$. Bulk material tested is CuO (purity 99.99\%) purchased also from Sigma-Aldrich. Tested CuO (Nano or Bulk) at a concentration of $200 \mathrm{mg}$. $\mathrm{L}^{-1}$ were sonicated for 30 minutes to ensure dispersion in the solution.This suspension was further diluted to obtain the remaining concentrations of $25,50,100,150$ and $200 \mathrm{mg}$. $\mathrm{L}^{-1}$.

\subsection{Phytotoxicity assay}

Phytotoxicity of previously prepared suspensions of $\mathrm{CuO}$ (Nano or Bulk) was tested using Maize seeds (Zea mays cv. hybrids triple white $\mathrm{Hi}$ Tech.). Well selected seeds were immersed in $10 \% \mathrm{H}_{2} \mathrm{O}_{2}$ solution for $10 \mathrm{~min}$ and rinsed thoroughly with deionized water to ensure surface sterility. The seeds were subsequently placed in Petri dishes $(100 \mathrm{~mm} \times 15 \mathrm{~mm}$ ); there were 10 seeds per Petri dish. Seeds germinated on filter paper moistened with $10 \mathrm{~mL}$ of tested metal oxide (Nano or Bulk) suspensions in final concentrations 25, 50, 100, 150 and $200 \mathrm{mg}^{-\mathrm{L}^{-1}}$. All experiments were performed in six replications. The seeds were covered in the dark at $24^{\circ} \mathrm{C}$. The length of roots and shoots were measured over the course of 8 days and compared to the untreated control. After measurement, whole seedlings were washed briefly with deionized water, in order to avoid washing out freshly acquired $\mathrm{Cu}^{+2}$, blotted gently with filter paper. The seedlings were quickly weighted for fresh weight determination, then oven-dried at $70^{\circ} \mathrm{C}$ for 48 hours in order to determine dry weight. The $\mathrm{Cu}$ content in tissues from all treatments was measured by Atomic Absorption Spectroscopy (AAS) after $\mathrm{HNO}_{3}$ digestion according to Sawhney and Frink (1991).

\subsection{Preparation of the extract}

Another fraction of fresh seedlings were immediately weighted and ground in a chilled mortar and pestle with $6 \mathrm{ml}$ buffer solution containing Tris $\mathrm{HCl} 0.05 \mathrm{M}, \mathrm{PH} 7.0$ consisting of $3 \mathrm{mM} \mathrm{MgCl}_{2}$ and $1 \mathrm{mM}$ EDTA. The extract was centrifuged at $4{ }^{\circ} \mathrm{C}$ for $10 \mathrm{~min}$ at $5000 \mathrm{rpm}$ and the supernatant obtained was used for the determination of enzymatic and non-enzymatic antioxidants and for the determination of antioxidant potential.

\subsection{Determination of photosynthetic pigments}

The photosynthetic pigments, via, chlorophyll a, chlorophyll $b$ and carotenoids, were extracted from fresh plumules samples. The plumules tissues were suspended in $5 \mathrm{ml}$ of $95 \%$ ethyl alcohol in a test tube at $60^{\circ} \mathrm{C}$, until colorless. Then the total volume completed into $10 \mathrm{ml}$ with $95 \%$ ethyl alcohol and absorbance readings were determined spectrophotometerically. Chlorophylls and carotenoids concentrations were calculated as $\mathrm{mg} \mathrm{g}^{-1} \mathrm{FW}$ at 663,644 and $452 \mathrm{~nm}$ using equations as cited by Lichtenthaler (1987).

\subsection{Determination of soluble proteins}

Protein contents were determined in the plant extract by Folin reagent according to Lowry et al. (1951). A calibration curve was constructed using bovine serum albumin (BSA) and the data were expressed as $\mathrm{mg} \mathrm{BSA}^{-1}$ fresh matter.

\subsection{Enzymatic antioxidants}

\subsubsection{Assay of superoxide dismutase}

SOD activity Determination carried out according to of Beauchamp and Fedovich (1976) method. SOD Unit was expressed as the amount of enzyme causing the reduction of NBT by $50 \%$. The expression of specific activity was in terms of units per $\mathrm{mg}$ of protein.

\subsubsection{Catalase assay}

CAT activity determination carried according to Aebi (1984). The decrease in $\mathrm{H}_{2} \mathrm{O}_{2}$ absorbance at $\mathrm{A} 240 \mathrm{~nm}$ was used to calculate the activity.

\subsubsection{Guaiacol peroxidase assay}

GPX activity determination carried out following the method of Tatiana et al. (1999). The increase in absorbance at $\mathrm{A} 470 \mathrm{~nm}$ due to the formation of tetraguaiacol was measured.

\subsubsection{Assay of ascorbate peroxidase}

APX activity was assayed following the method of Jiang and Zhang (2002). The decrease in A290 following the oxidation rate of ascorbic acid was measured.

\subsubsection{Assay of lipoxygenase}

The method of Minguez-Mosquera et al. (1993) was modified and used to assay lipoxygenase activity. The substrate was prepared by solubilizing $0.5 \mathrm{~g}$ linoleic acid with $0.5 \mathrm{~g}$ Tween 20 in deionized water and the final volume brought to $25 \mathrm{ml}$. Turbidity was cleared with a few drops of $2 \mathrm{~N} \mathrm{NaOH}$. The plant extract was reacted with the substrate in a spectrophotometer cuvette containing $3 \mathrm{ml}$ phosphate buffer $0.2 \mathrm{M}$, at pH 6.5 and the absorbance 
measured at $234 \mathrm{~nm}$ at $20 \mathrm{~s}$ intervals for $1 \mathrm{~min}$ using a recording spectrophotometer. The rate of formation of conjugated diene reaction products, measured as an increase in A234 nm.

\subsection{Determination of non-enzymatic antioxidants}

\subsubsection{Total phenolics determination}

Total phenolic contents were assessed according to Singleton and Rossi (1965). Folin-Ciocalteau reagent method was used. The measurements carried out at A765 $\mathrm{nm}$. Gallic acid equivalents were used to express the data as $\mu \mathrm{g} \mathrm{g}^{-1} \mathrm{FW}$ using Molar Coefficient of $120 \mathrm{\mu g}^{-1} \mathrm{~cm}^{-1} \mathrm{ml}^{-1}$.

\subsubsection{Total flavonoids determination}

Content of total flavonoid was measured according to Moreno et al. (2000). Quercetin equivalents were used to express the absorbance at $\mathrm{A} 415 \mathrm{~nm}$ as $\mathrm{mg} \mathrm{g}^{-1} \mathrm{FW}$.

\subsection{Antioxidant ability assays}

\subsubsection{Total antioxidant activity}

The total antioxidant contents were estimated following the method of Prieto et al. (1999). $0.1 \mathrm{ml}$ of the plant extract was combined with $3 \mathrm{ml}$ of the reagent solution (0.6 $\mathrm{M} \mathrm{H}_{2} \mathrm{SO}_{4}, 28 \mathrm{mMNa}_{3} \mathrm{PO}_{4}$ and $4 \mathrm{mM}$ ammonium molybdate); the mixture was incubated at $95{ }^{\circ} \mathrm{C}$ for 90 minutes and then cooled to room temperature. The absorbance was measured at $695 \mathrm{~nm}$.

\subsubsection{Reducing power assay}

The reducing power of the samples was determined according to the procedure described by Oyaizu (1986) with modifications. $0.1 \mathrm{ml}$ of the plant extractwas mixed with $0.5 \mathrm{ml}$ of $0.2 \mathrm{M}$ phosphate buffer $(\mathrm{pH}=6.6)$ and $0.5 \mathrm{ml}$ of $0.1 \% \mathrm{~K}_{3}\left[\mathrm{Fe}(\mathrm{CN})_{3}\right]$; the mixture was incubated in a water bath for 20 minutes at 50 ㅇ. After adding $0.5 \mathrm{ml}$ trichloroacetic acid, the mixture was centrifuged at $1000 \mathrm{rpm}$ for 10 minutes. To the supernatant $(1 \mathrm{ml}), 1 \mathrm{ml}$ distilled waterand $100 \mu \mathrm{l}$ of $0.01 \% \mathrm{FeCl}_{3}$ were added. The mixture was then incubated at $37 \stackrel{\circ}{\circ}$ for 10 minutes; after which, the absorbance was measured at $700 \mathrm{~nm}$. Ascorbic acid solution was used to construct a standard curve. The results were expressed as ascorbic acid equivalents as $\mu \mathrm{g} \mathrm{g}^{-1}$ fresh matter.

\subsubsection{Hydroxyl radical $\left(\mathrm{OH}^{-}\right)$scavenging assay}

$\mathrm{OH}^{-}$radical scavenging assay carried out according to Kunchandy and Rao (1990). Absorbance of plant extract was measured against a blank containing deoxyribose and buffer at $\mathrm{A} 532 \mathrm{~nm}$, and degradation inhibition of deoxyribose was used to calculate the inhibition in percent (I) was calculated by the formula $I=$ (Abs control - Abs sample)/Abs control $\times 100$.

\subsubsection{Hydrogen peroxide $\left(\mathrm{H}_{2} \mathrm{O}_{2}\right)$ scavenging}

$\mathrm{H}_{2} \mathrm{O}_{2}$ radical scavenging assay carried out according to Long et al. (1999). Sodium pyruvate was used as the reference compound. The absorbance of the ferric-xylenol orange complex was measured at A560 nm.

\subsubsection{Lipid peroxide formation inhibition}

Lipid peroxidation inhibition\% carried out according to Janero (1990). The absorbance of the upper organic layer was measured at A532 $\mathrm{nm}$. The inhibition in percent (I) was calculated by the formula $I=$ (Abs control - Abs sample)/Abs control $\times 100$.

\subsubsection{Metal chelating assay}

Metal chelating ability carried out according to Decker and Barbara (1990). The absorbance of the solution was measured at A562 $\mathrm{nm}$. EDTA was used as a positive control.

\subsection{Statistical analysis}

For all the experiments (complete randomized design) three samples were analyzed and all the assays were carried out in triplicate. All values described in results section were mean of three replications \pm standard error. Analysis of variance (ANOVA) was carried out using SPSS v16.0, followed by Duncan's multiple range test between the means of treatments to determine the significant difference at the probability level $<0.05$. All the assessed attributes subjected to cluster analysis using a Correlations similarity distance with the software PAST version 2.11 for Windows (Hammer et al., 2001). The matrix was then analyzed with Principle Component Analysis (PCA) variance regression ordination, using the Sørensen coefficient as the distance measure, to check the magnitude of change in attributes along the $\mathrm{CuO}$ and CuONP gradients by the same software.

\section{Results}

Copper, as a heavy metal, stress caused a significant decrease in most of the investigated parameters and it unfortunately inter to the plant body in exponential manner by the increase of its concentration in the circumstance. In this respect, $\mathrm{Cu}$ reached to $260 \mathrm{\mu g} \mathrm{g}^{-1} \mathrm{DW}$ in the plants grown in $200 \mathrm{mg} \mathrm{L}^{-1} \mathrm{CuO}$ and $82 \mathrm{\mu g} \mathrm{g}^{-1} \mathrm{DW}$ in the plants grown in $100 \mathrm{mg} \mathrm{L}^{-1}$ CuONP (Table 1). Generally, the maize growth was completely stopped under 150 and $200 \mathrm{mgL}^{-1}$ CuONP. Maize seedling fresh and dry weight and shoot and root lengthsdecreased gradually in bulk-conditions till the minimum at $200 \mathrm{mgL}^{-1}$ (Table 1).However, at Nano-conditions; the same effect was observed for all CuONP concentrations till $100 \mathrm{mgL}^{-1}$ that induced the same responseofits twofold of bulk-conditions. Water content also decreased significantly by increase in $\mathrm{CuO}$ and CuONP concentrations (Table 1).

Plumule pigments showed somewhat different trends toward the different concentrations of both $\mathrm{CuO}$ and CuONP. Chlorophyll a and b content decreased in bulk Cuat 25, 50 and $100 \mathrm{mg} \mathrm{L}^{-1}$ and nano-Cu at 25 and $50 \mathrm{mg} \mathrm{L}^{-1}$ for about the half of control (no Copper) with no significant difference between them. Other significant decrease was recorded in $150 \mathrm{mg} \mathrm{L}^{-1}$ followed by slight increase in $200 \mathrm{mg} \mathrm{L}^{-1}$ under bulk-conditions and reached to the quadrate of the control at the last two bulk concentrations (150 and $\left.200 \mathrm{mg} \mathrm{L}^{-1}\right)$. Differences in chlorophyll b between 25, 50 and $100 \mathrm{mg} \mathrm{L}^{-1}$ can be neglected with the unusual significant increase under 100 CuONP which statistically equal to the control.Furthermore, carotenoids follow the same steps with notable peak at $100 \mathrm{mg} \mathrm{L}^{-1}$ for both conditions (Table 1). 
Table 1. Growth attributes and copper content of (Zea mays cv. hybrids triple white Hi Tech.) as influenced by the toxicity of bulk-CuO and nano-CuO (mean $\pm \mathrm{SE} ; \mathrm{n}=3$ )

\begin{tabular}{|c|c|c|c|c|c|c|c|c|c|c|}
\hline $\begin{array}{c}\mathrm{Cu} \\
\text { Conc. } \\
\mathrm{mg} \mathrm{L}^{-1}\end{array}$ & & $\begin{array}{c}\mathrm{Cu} \\
\mu \mathrm{g} \mathrm{g}^{-1} \\
\mathrm{DW}\end{array}$ & $\begin{array}{l}\mathrm{FW} \\
\mathrm{mg}\end{array}$ & $\begin{array}{l}\text { DW } \\
\mathrm{mg}\end{array}$ & WC\% & $\begin{array}{l}\text { Shoot } \\
\text { Length } \\
\text { (mm.) }\end{array}$ & $\begin{array}{l}\text { Root Length } \\
\text { (mm.) }\end{array}$ & $\begin{array}{c}\text { Chlorophy } \\
\text { II a } \\
\mathrm{mg} \mathrm{g}^{-1} \mathrm{FW}\end{array}$ & $\begin{array}{c}\text { Chlorophyll } \\
\text { b } \\
\mathrm{mg} \mathrm{g}^{-1} \mathrm{FW}\end{array}$ & $\begin{array}{c}\text { Caroten } \\
\text { oids } \\
\text { mg g }^{-1} \\
\text { FW }\end{array}$ \\
\hline Control & Zero & $\begin{array}{c}10 \pm \\
1.11^{\mathrm{a}} \\
\end{array}$ & $\begin{array}{c}369 \pm \\
4.5^{i} \\
\end{array}$ & $\begin{array}{c}33.20 \pm \\
0.57^{i} \\
\end{array}$ & $\begin{array}{c}91.0 \pm \\
2.5^{c} \\
\end{array}$ & $\begin{array}{c}54.67 \pm \\
1.42^{d} \\
\end{array}$ & $\begin{array}{c}83.33 \pm \\
3.02^{d} \\
\end{array}$ & $\begin{array}{c}109.43 \pm \\
1.49^{\mathrm{d}} \\
\end{array}$ & $\begin{array}{c}20.06 \pm \\
0.07^{\text {cd }} \\
\end{array}$ & $\begin{array}{c}52.82 \pm \\
0.94^{\mathrm{g}} \\
\end{array}$ \\
\hline \multirow{5}{*}{$\begin{array}{l}\text { Bulk } \\
\text { (CuO) }\end{array}$} & 25 & $\begin{array}{c}14.2 \pm \\
1.02^{\mathrm{b}} \\
\end{array}$ & $\begin{array}{c}363 \pm \\
2.9^{\mathrm{h}} \\
\end{array}$ & $\begin{array}{c}32.17 \pm \\
0.39^{\mathrm{h}} \\
\end{array}$ & $\begin{array}{c}91.1 \pm \\
2.9^{\mathrm{d}} \\
\end{array}$ & $\begin{array}{c}35.33 \pm \\
1.21^{\mathrm{c}} \\
\end{array}$ & $21.67 \pm 1.34^{c}$ & $\begin{array}{c}44.21 \pm \\
1.21^{\mathrm{c}} \\
\end{array}$ & $\begin{array}{l}12.35 \pm \\
0.30^{\mathrm{abc}} \\
\end{array}$ & $\begin{array}{c}26.03 \pm \\
0.53^{c} \\
\end{array}$ \\
\hline & 50 & $\begin{array}{c}18.5 \pm \\
1.41^{c} \\
\end{array}$ & $\begin{array}{c}249 \pm \\
7.3^{\mathrm{e}} \\
\end{array}$ & $\begin{array}{c}27.07 \pm \\
0.45^{\mathrm{g}} \\
\end{array}$ & $\begin{array}{c}89.1 \pm \\
1.9^{\mathrm{b}} \\
\end{array}$ & $\begin{array}{c}34.00 \pm \\
1.21^{\mathrm{c}} \\
\end{array}$ & $\begin{array}{l}11.67 \pm \\
0.97^{\mathrm{abc}}\end{array}$ & $\begin{array}{c}40.13 \pm \\
0.96^{c} \\
\end{array}$ & $\begin{array}{l}13.57 \pm \\
0.03^{\text {abcd }}\end{array}$ & $\begin{array}{c}30.20 \pm \\
0.80^{d} \\
\end{array}$ \\
\hline & 100 & $\begin{array}{c}49.8 \pm \\
4.01^{f} \\
\end{array}$ & $\begin{array}{c}240 \pm \\
3.5^{d} \\
\end{array}$ & $\begin{array}{c}20.93 \pm \\
0.51^{\mathrm{e}} \\
\end{array}$ & $\begin{array}{c}91.3 \pm \\
3.5^{\mathrm{e}} \\
\end{array}$ & $\begin{array}{c}20.00 \pm \\
1.89^{\mathrm{ab}} \\
\end{array}$ & $6.67 \pm 1.02^{\mathrm{ab}}$ & $\begin{array}{c}42.65 \pm \\
4.13^{c} \\
\end{array}$ & $\begin{array}{l}14.21 \pm \\
0.94^{\text {bcd }} \\
\end{array}$ & $\begin{array}{c}37.31 \pm \\
0.45^{f} \\
\end{array}$ \\
\hline & 150 & $\begin{array}{l}70 \pm \\
3.1^{\mathrm{g}} \\
\end{array}$ & $\begin{array}{c}226 \pm \\
2.1^{c} \\
\end{array}$ & $\begin{array}{c}18.33 \pm \\
0.67^{c} \\
\end{array}$ & $\begin{array}{c}91.9 \pm \\
2.8^{f} \\
\end{array}$ & $\begin{array}{c}16.67 \pm \\
1.67^{\mathrm{ab}} \\
\end{array}$ & $3.00 \pm 0.58^{\mathrm{a}}$ & $\begin{array}{c}20.81 \pm \\
0.75^{\mathrm{a}} \\
\end{array}$ & $6.05 \pm 0.75^{a}$ & $\begin{array}{c}14.63 \pm \\
0.35^{\mathrm{a}} \\
\end{array}$ \\
\hline & 200 & $\begin{array}{r}260 \pm \\
5.21^{i} \\
\end{array}$ & $\begin{array}{l}206 \pm \\
3.29^{b}\end{array}$ & $\begin{array}{l}6.33 \pm \\
0.44^{\mathrm{a}} \\
\end{array}$ & $\begin{array}{c}96.9 \pm \\
4.1^{i} \\
\end{array}$ & $\begin{array}{c}15.00 \pm \\
1.70^{\mathrm{ab}}\end{array}$ & $2.00 \pm 0.00^{\mathrm{a}}$ & $\begin{array}{c}28.53 \pm \\
0.89^{\mathrm{b}}\end{array}$ & $\begin{array}{l}6.82 \pm \\
0.35^{\mathrm{ab}}\end{array}$ & $\begin{array}{c}16.73 \pm \\
0.58^{\mathrm{b}}\end{array}$ \\
\hline \multirow{3}{*}{$\begin{array}{c}\text { Nano } \\
\text { (CuONP } \\
\text { ) }\end{array}$} & 25 & $20 \pm 3^{d}$ & $\begin{array}{l}320 \pm \\
3.08^{\mathrm{g}}\end{array}$ & $\begin{array}{c}21.53 \pm \\
0.29^{f}\end{array}$ & $\begin{array}{c}93.4 \pm \\
3.4^{\mathrm{h}} \\
\end{array}$ & $\begin{array}{c}23.33 \pm \\
1.33^{\mathrm{b}}\end{array}$ & $\begin{array}{c}18.33 \pm \\
1.33^{b c}\end{array}$ & $\begin{array}{c}42.54 \pm \\
4.02^{\mathrm{C}} \\
\end{array}$ & $\begin{array}{l}12.69 \pm \\
0.40^{\mathrm{abc}} \\
\end{array}$ & $\begin{array}{c}31.64 \pm \\
0.56^{\mathrm{e}}\end{array}$ \\
\hline & 50 & $\begin{array}{r}27.33 \\
\pm 2.14^{\mathrm{e}} \\
\end{array}$ & $\begin{array}{l}289 \pm \\
1.33^{f} \\
\end{array}$ & $\begin{array}{c}20.17 \pm \\
0.46^{d} \\
\end{array}$ & $\begin{array}{c}93.1 \pm \\
2.8^{\mathrm{g}} \\
\end{array}$ & $\begin{array}{c}16.67 \pm \\
1.67^{\mathrm{ab}} \\
\end{array}$ & $\begin{array}{l}10.00 \pm \\
0.00^{\mathrm{abc}}\end{array}$ & $\begin{array}{c}43.49 \pm \\
1.16^{c} \\
\end{array}$ & $\begin{array}{l}13.91 \pm \\
0.74^{\text {bcd }}\end{array}$ & $\begin{array}{c}29.41 \pm \\
0.55^{d} \\
\end{array}$ \\
\hline & 100 & $\begin{array}{l}82 \pm \\
3.2^{h}\end{array}$ & $\begin{array}{l}74 \pm \\
3.41^{a}\end{array}$ & $\begin{array}{c}12.50 \pm \\
0.66^{\mathrm{b}}\end{array}$ & $\begin{array}{c}83.1 \pm \\
2.9^{\mathrm{a}} \\
\end{array}$ & $\begin{array}{c}9.67 \pm \\
0.63^{\mathrm{a}} \\
\end{array}$ & $2.00 \pm 0.00^{\mathrm{a}}$ & $\begin{array}{c}41.88 \pm \\
3.56^{c}\end{array}$ & $\begin{array}{c}20.52 \pm \\
0.50^{d}\end{array}$ & $\begin{array}{c}36.03 \pm \\
0.74^{f}\end{array}$ \\
\hline
\end{tabular}

Values with same alphabets in superscript in a column do not differ significantly.

On the other hand, many pivotal primary (soluble proteins), secondary metabolites (total phenolics, total flavonoids) in addition to total antioxidants and reducing power showed counter response of the growth and pigments. All of them, in comparison to control, recorded significant increase under both $\mathrm{CuO}$ conditions. There was

Table 2. Biochemical attributes of (Zea mays cv. hybrids triple white $\mathrm{Hi}$ Tech.) as influenced by the toxicity of bulk-CuO and nano-CuO (mean $\pm \mathrm{SE} ; \mathrm{n}=3$ ) a general gradual increase in soluble protein contents under bulk-conditions till $150 \mathrm{mg} \mathrm{L}^{-1} \mathrm{CuO}$ while CuONP caused drastic jump at $25 \mathrm{mg} \mathrm{L}^{-1}$ (double of the control). Comparing the two conditions show the ferocity of $25 \mathrm{mg}$ $\mathrm{L}^{-1}$ CuONP that caused the same effect of $150 \mathrm{mg} \mathrm{L}^{-1} \mathrm{CuO}$ on soluble proteins.

\begin{tabular}{|c|c|c|c|c|c|c|c|c|c|c|}
\hline \multirow{2}{*}{\multicolumn{2}{|c|}{$\begin{array}{c}\text { Cu Conc. } \\
\mathrm{mg} \mathrm{L}^{-1}\end{array}$}} & \multirow{3}{*}{$\begin{array}{c}\text { Proteins } \\
\mathrm{mg} \mathrm{g}^{-1} \\
\text { FW } \\
\\
46.86 \pm \\
2.11^{\mathrm{a}} \\
\end{array}$} & \multirow{3}{*}{$\begin{array}{c}\text { Phenoli } \\
\text { c } \\
\mu \mathrm{g} \mathrm{g}^{-1} \\
\mathrm{FW} \\
2.08 \pm \\
0.10^{\mathrm{a}} \\
\end{array}$} & \multirow{3}{*}{$\begin{array}{l}\text { Flavonoid } \\
\begin{array}{c}\mathrm{s}^{-1} \\
\mathrm{mg} \mathrm{g}^{-1} \mathrm{FW}\end{array} \\
0.86 \pm \\
0.02^{\mathrm{a}} \\
\end{array}$} & \multirow{3}{*}{ 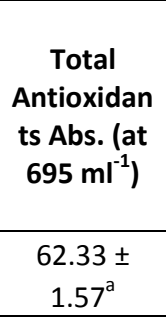 } & \multirow{3}{*}{$\begin{array}{c}\text { Lipid } \\
\text { Peroxida } \\
\text { tion } \\
\text { inhibitio } \\
\text { n \%. } \\
91.16 \pm \\
1.17^{\mathrm{e}}\end{array}$} & \multirow{3}{*}{$\begin{array}{c}\text { Hydroxyl } \\
\text { Radical } \\
\text { Scavengi } \\
\text { ng \% } \\
94.96 \pm \\
1.47^{\ddagger} \\
\end{array}$} & \multirow{3}{*}{$\begin{array}{c}\begin{array}{c}\text { Reducin } \\
\mathrm{g} \mathrm{Power}^{-1} \\
\boldsymbol{\mu g} \mathbf{g}^{-1} \\
\text { FW }\end{array} \\
0.23 \pm \\
0.01^{\mathrm{a}}\end{array}$} & \multirow{3}{*}{$\begin{array}{c}\mathrm{H}_{2} \mathrm{O}_{2} \\
\text { Radical } \\
\text { Scavengi } \\
\text { ng \% }\end{array}$} & \multirow{3}{*}{$\begin{array}{c}\begin{array}{c}\text { Metal } \\
\text { chelatir } \\
\text { g \% }\end{array} \\
93.20 \pm \\
1.38^{i} \\
\end{array}$} \\
\hline & & & & & & & & & & \\
\hline $\begin{array}{c}\text { Cont } \\
\text { rol }\end{array}$ & Zero & & & & & & & & & \\
\hline \multirow{5}{*}{$\begin{array}{c}\text { Bulk } \\
\text { (CuO } \\
\text { ) }\end{array}$} & 25 & $\begin{array}{c}80.67 \pm \\
1.10^{\mathrm{d}}\end{array}$ & $\begin{array}{l}3.05 \pm \\
0.08^{c}\end{array}$ & $\begin{array}{l}1.80 \pm \\
0.05^{c}\end{array}$ & $\begin{array}{c}101.54 \pm \\
0.95^{\text {ef }}\end{array}$ & $\begin{array}{c}89.88 \pm \\
0.96^{\mathrm{e}}\end{array}$ & $\begin{array}{c}93.19 \pm \\
0.96^{\mathrm{h}}\end{array}$ & $\begin{array}{l}0.30 \pm \\
0.01^{\mathrm{b}}\end{array}$ & $\begin{array}{c}96.32 \pm \\
1.19^{\mathrm{h}}\end{array}$ & $\begin{array}{c}92.01 \pm \\
1.28^{\mathrm{h}}\end{array}$ \\
\hline & 50 & $\begin{array}{c}90.77 \pm \\
1.22^{\mathrm{e}}\end{array}$ & $\begin{array}{l}2.66 \pm \\
0.06^{b}\end{array}$ & $\begin{array}{l}2.05 \pm \\
0.05^{d}\end{array}$ & $\begin{array}{c}100.36 \pm \\
3.04^{\text {def }}\end{array}$ & $\begin{array}{c}83.36 \pm \\
1.19^{d}\end{array}$ & $\begin{array}{c}90.20 \pm \\
1.96^{\mathrm{e}}\end{array}$ & $\begin{array}{l}0.38 \pm \\
0.01^{c}\end{array}$ & $\begin{array}{c}96.08 \pm \\
0.29^{\mathrm{g}}\end{array}$ & $\begin{array}{c}86.27 \pm \\
0.56^{f}\end{array}$ \\
\hline & 100 & $\begin{array}{c}72.95 \pm \\
2.29^{c}\end{array}$ & $\begin{array}{l}3.22 \pm \\
0.04^{\mathrm{cd}}\end{array}$ & $\begin{array}{l}1.35 \pm \\
0.04^{b}\end{array}$ & $\begin{array}{c}108.17 \pm \\
2.12^{f}\end{array}$ & $\begin{array}{c}83.96 \pm \\
0.95^{\mathrm{d}}\end{array}$ & $\begin{array}{c}89.17 \pm \\
1.16^{d}\end{array}$ & $\begin{array}{l}0.44 \pm \\
0.02^{d}\end{array}$ & $\begin{array}{c}92.05 \pm \\
1.36^{\mathrm{c}}\end{array}$ & $\begin{array}{c}82.60 \pm \\
1.81^{d}\end{array}$ \\
\hline & 150 & $\begin{array}{c}109.12 \pm \\
2.4^{f}\end{array}$ & $\begin{array}{l}3.71 \pm \\
0.03^{\mathrm{e}}\end{array}$ & $\begin{array}{l}1.46 \pm \\
0.05^{b}\end{array}$ & $\begin{array}{c}95.88 \pm \\
1.19^{\mathrm{de}}\end{array}$ & $\begin{array}{c}66.36 \pm \\
2.44^{\mathrm{b}}\end{array}$ & $\begin{array}{c}78.26 \pm \\
0.98^{\mathrm{a}}\end{array}$ & $\begin{array}{c}0.75 \pm \\
0.02^{\mathrm{g}}\end{array}$ & $\begin{array}{c}88.77 \pm \\
1.19^{\mathrm{a}}\end{array}$ & $\begin{array}{c}72.17 \pm \\
1.25^{\mathrm{a}}\end{array}$ \\
\hline & 200 & $\begin{array}{c}93.93 \pm \\
1.32^{\mathrm{e}}\end{array}$ & $\begin{array}{l}2.77 \pm \\
0.04^{b}\end{array}$ & $\begin{array}{l}1.84 \pm \\
0.05^{\text {cd }}\end{array}$ & $\begin{array}{c}78.75 \pm \\
0.94^{\mathrm{b}}\end{array}$ & $\begin{array}{c}74.10 \pm \\
1.21^{\mathrm{c}}\end{array}$ & $\begin{array}{c}86.01 \pm \\
1.37^{c}\end{array}$ & $\begin{array}{l}0.56 \pm \\
0.01^{\mathrm{e}}\end{array}$ & $\begin{array}{c}93.88 \pm \\
1.47^{d}\end{array}$ & $\begin{array}{c}79.11 \pm \\
1.28^{c}\end{array}$ \\
\hline \multirow{3}{*}{$\begin{array}{l}\text { Nano } \\
\text { (CuO } \\
\text { NP) }\end{array}$} & 25 & $\begin{array}{c}109.47 \pm \\
0.82^{f}\end{array}$ & $\begin{array}{l}3.38 \pm \\
0.08^{d} \\
\end{array}$ & $\begin{array}{l}1.98 \pm \\
0.08^{\text {cd }}\end{array}$ & $\begin{array}{c}99.01 \pm \\
2.56^{\text {def }}\end{array}$ & $\begin{array}{c}83.53 \pm \\
1.99^{\mathrm{d}}\end{array}$ & $\begin{array}{c}91.04 \pm \\
0.85^{\mathrm{g}} \\
\end{array}$ & $\begin{array}{l}0.46 \pm \\
0.02^{d} \\
\end{array}$ & $\begin{array}{c}95.14 \pm \\
1.00^{\mathrm{e}}\end{array}$ & $\begin{array}{c}85.40 \pm \\
0.45^{\mathrm{e}}\end{array}$ \\
\hline & 50 & $\begin{array}{c}65.40 \pm \\
1.2^{b}\end{array}$ & $\begin{array}{l}3.26 \pm \\
0.06^{d} \\
\end{array}$ & $\begin{array}{l}1.91 \pm \\
0.05^{\text {cd }}\end{array}$ & $\begin{array}{c}85.40 \pm \\
2.74^{\text {bc }}\end{array}$ & $\begin{array}{c}85.80 \pm \\
0.72^{d}\end{array}$ & $\begin{array}{c}90.59 \pm \\
1.18^{f} \\
\end{array}$ & $\begin{array}{c}0.40 \pm \\
0.01^{c} \\
\end{array}$ & $\begin{array}{c}95.77 \pm \\
0.93^{f}\end{array}$ & $\begin{array}{c}89.63 \pm \\
1.12^{\mathrm{g}}\end{array}$ \\
\hline & 100 & $\begin{array}{c}65.27 \pm \\
0.86^{\mathrm{b}}\end{array}$ & $\begin{array}{l}3.05 \pm \\
0.03^{c}\end{array}$ & $\begin{array}{l}2.35 \pm \\
0.04^{\mathrm{e}}\end{array}$ & $\begin{array}{c}89.86 \pm \\
1.28^{\text {cd }}\end{array}$ & $\begin{array}{c}61.81 \pm \\
1.41^{\mathrm{a}}\end{array}$ & $\begin{array}{c}81.58 \pm \\
1.13^{\mathrm{b}}\end{array}$ & $\begin{array}{c}0.64 \pm \\
0.01^{f}\end{array}$ & $\begin{array}{c}89.76 \pm \\
1.10^{\mathrm{b}}\end{array}$ & $\begin{array}{c}78.85 \pm \\
0.97^{\mathrm{b}}\end{array}$ \\
\hline
\end{tabular}

Values with same alphabets in superscript in a column do not differ significantly. 
Overall ability of the cell to detoxify ROS was measured by total antioxidants activity and reducing power. Zea mays seedlings raised their reducing power agents under bulkconditions until they reachedthe maximum (about 3.5 folds of control) at $150 \mathrm{mg} \mathrm{L}^{-1} \mathrm{CuO}$ while it turned to

Table 3. activities of lipoxygenase and some enzymatic antioxidants of (Zea mays cv. hybrids triple white Hi Tech.) as influenced by the toxicity of bulk-CuO and nano-CuO (mean $\pm \mathrm{SE} ; \mathrm{n}=3$ )

\begin{tabular}{|c|c|c|c|c|c|c|}
\hline \multicolumn{2}{|c|}{ Cu Conc. $\mathrm{mg} \mathrm{L}^{-1}$} & \multirow{2}{*}{$\begin{array}{c}\begin{array}{c}\text { LOX min }_{\text {mg }}{ }^{-1} \\
\text { proteins }\end{array} \\
1.89 \pm 0.07^{\mathrm{b}} \\
\end{array}$} & \multirow{2}{*}{$\begin{array}{c}\begin{array}{c}\text { SOD unit } \mathrm{mg}^{-1} \\
\text { proteins }\end{array} \\
2.07 \pm 0.14^{\mathrm{a}} \\
\end{array}$} & \multirow{2}{*}{$\begin{array}{c}\text { POD min } \mathrm{mg}^{-1} \\
\text { proteins }\end{array}$} & \multirow{2}{*}{$\begin{array}{c}\begin{array}{c}\text { CAT min } \mathrm{mg}^{-1} \\
\text { proteins }\end{array} \\
0.05 \pm 0.003^{\mathrm{C}}\end{array}$} & \multirow{2}{*}{$\begin{array}{c}\begin{array}{c}\mathrm{APX} \min \mathrm{mg}^{-1} \\
\text { proteins }\end{array} \\
0.04 \pm 0.003^{\mathrm{C}}\end{array}$} \\
\hline Control & Zero & & & & & \\
\hline & 25 & $3.82 \pm 0.10^{d}$ & $2.28 \pm 0.14^{a}$ & $0.04 \pm 0.001^{c}$ & $0.04 \pm 0.001^{a}$ & $0.04 \pm 0.002^{\mathrm{e}}$ \\
\hline & 50 & $2.78 \pm 0.14^{c}$ & $2.16 \pm 0.33^{\mathrm{a}}$ & $0.04 \pm 0.001^{\mathrm{e}}$ & $0.06 \pm 0.001^{f}$ & $0.03 \pm 0.002^{a}$ \\
\hline \multirow[t]{4}{*}{ Bulk (CuO) } & 100 & $4.41 \pm 0.05^{\mathrm{e}}$ & $4.45 \pm 0.32^{b c}$ & $0.07 \pm 0.001^{h}$ & $0.08 \pm 0.001^{g}$ & $0.07 \pm 0.002^{\mathrm{g}}$ \\
\hline & 150 & $5.56 \pm 0.14^{f}$ & $9.21 \pm 0.17^{d}$ & $0.06 \pm 0.005^{f}$ & $0.06 \pm 0.002^{\mathrm{e}}$ & $0.05 \pm 0.004^{f}$ \\
\hline & 200 & $1.34 \pm 0.13^{\mathrm{a}}$ & $5.43 \pm 0.32^{c}$ & $0.04 \pm 0.001^{d}$ & $0.08 \pm 0.001^{h}$ & $0.08 \pm 0.002^{h}$ \\
\hline & 25 & $2.07 \pm 0.09^{b}$ & $2.32 \pm 0.20^{a}$ & $0.02 \pm 0.003^{\mathrm{a}}$ & $0.04 \pm 0.003^{b}$ & $0.03 \pm 0.002^{b}$ \\
\hline \multirow[t]{2}{*}{ Nano (CuONP) } & 50 & $3.60 \pm 0.07^{d}$ & $3.42 \pm 0.36^{\mathrm{ab}}$ & $0.07 \pm 0.002^{g}$ & $0.05 \pm 0.003^{d}$ & $0.04 \pm 0.002^{d}$ \\
\hline & 100 & $3.81 \pm 0.12^{d}$ & $15.82 \pm 0.50^{\mathrm{e}}$ & $0.07 \pm 0.003^{i}$ & $0.15 \pm 0.003^{i}$ & $0.15 \pm 0.003^{i}$ \\
\hline
\end{tabular}

Values with same alphabets in superscript in a column do not differ significantly

Total antioxidants made an arch with the maximum at 100 $\mathrm{mg} \mathrm{L}^{-1} \mathrm{CuO}$ while this peak was recorded at $25 \mathrm{mg} \mathrm{L}^{-1}$ CuONP which statistically equal to the response under 100 $\mathrm{mg} \mathrm{L}^{-1} \mathrm{CuO}$. Almost the same arch was representedin the response of total phenolic compounds while the significant increase continued for $150 \mathrm{mg} \mathrm{L}^{-1} \mathrm{CuO}$.

Table 4. Correlation coefficient values ( $\mathrm{r} 2$ ) among different parameters of Zea mays as affected by different CuO concentrations under bulk and nano-conditions

\begin{tabular}{|c|c|c|c|c|c|c|c|c|c|c|c|c|c|c|c|c|c|c|c|c|c|c|}
\hline & FW & DW & $\begin{array}{l}\text { w } \\
\text { C }\end{array}$ & SH & $\mathbf{R L}$ & $\begin{array}{c}\mathrm{Ch}_{-} \\
\mathrm{a}\end{array}$ & $\underset{\mathrm{b}}{\mathrm{Ch}}$ & $\begin{array}{l}\mathrm{Ca} \\
\text { rot }\end{array}$ & $\mathbf{P h}$ & $\begin{array}{l}R \\
P\end{array}$ & $\begin{array}{l}\mathbf{T} \\
\mathbf{A}\end{array}$ & $\begin{array}{l}F \\
\text { I }\end{array}$ & $\begin{array}{l}\mathbf{P} \\
\mathbf{r} \\
\mathbf{0}\end{array}$ & $\begin{array}{l}\mathbf{L} \\
\mathbf{P}\end{array}$ & $\begin{array}{l}\mathbf{H} \\
\mathrm{R}\end{array}$ & $\begin{array}{l}\mathrm{H}_{2} \\
\mathrm{O}_{2}\end{array}$ & $\begin{array}{c}\mathrm{M} \\
\mathrm{C}\end{array}$ & $\begin{array}{l}\mathrm{L} \\
\mathbf{O} \\
\mathrm{X}\end{array}$ & $\begin{array}{l}\text { S } \\
\text { O } \\
\text { D }\end{array}$ & $\begin{array}{l}\mathbf{P} \\
\mathbf{0} \\
\mathbf{D}\end{array}$ & $\begin{array}{l}\mathbf{C} \\
\mathbf{A} \\
\mathbf{T}\end{array}$ & $\begin{array}{l}\mathbf{A} \\
\mathbf{P} \\
\mathbf{X}\end{array}$ \\
\hline $\mathrm{F}$ & 1 & & & & & & & & & & & & & & & & & & & & & \\
\hline \multicolumn{23}{|l|}{ w } \\
\hline $\mathrm{D}$ & 0.94 & 1 & & & & & & & & & & & & & & & & & & & & \\
\hline W & $* * *$ & & & & & & & & & & & & & & & & & & & & & \\
\hline $\mathrm{W}$ & 0.77 & 0.6 & 1 & & & & & & & & & & & & & & & & & & & \\
\hline $\mathrm{C}$ & $*$ & $8^{*}$ & & & & & & & & & & & & & & & & & & & & \\
\hline \multirow[t]{2}{*}{$\mathrm{SH}$} & 0.87 & 0.8 & 0. & 1 & & & & & & & & & & & & & & & & & & \\
\hline & $* *$ & $5 * *$ & 62 & & & & & & & & & & & & & & & & & & & \\
\hline \multirow[t]{2}{*}{$\mathrm{RL}$} & 0.71 & 0.5 & 0. & 0.8 & 1 & & & & & & & & & & & & & & & & & \\
\hline & $*$ & 9 & 56 & $9 * *$ & & & & & & & & & & & & & & & & & & \\
\hline $\mathrm{Ch}$ & 0.65 & 0.4 & 0. & 0.8 & 0.96 & 1 & & & & & & & & & & & & & & & & \\
\hline${ }_{-}^{a}$ & & 9 & 46 & $2 * *$ & $* * *$ & & & & & & & & & & & & & & & & & \\
\hline $\mathrm{Ch}$ & 0.37 & 0.1 & 0. & 0.3 & 0.51 & 0.7 & 1 & & & & & & & & & & & & & & & \\
\hline _b & & 7 & 01 & 6 & & $0 *$ & & & & & & & & & & & & & & & & \\
\hline $\mathrm{Ca}$ & 0.54 & 0.3 & 0. & 0.6 & 0.75 & 0.8 & 0.90 & 1 & & & & & & & & & & & & & & \\
\hline rot & & 6 & 27 & 1 & $*$ & $9 * *$ & $* * *$ & & & & & & & & & & & & & & & \\
\hline \multirow[t]{3}{*}{$\mathrm{Ph}$} & - & - & - & - & - & - & - & - & 1 & & & & & & & & & & & & & \\
\hline & 0.40 & 0.3 & .1 & 0.7 & 0.72 & 0.7 & 0.50 & 0.6 & & & & & & & & & & & & & & \\
\hline & & 6 & 5 & $2^{*}$ & $*$ & 7* & & 2 & & & & & & & & & & & & & & \\
\hline \multirow[t]{3}{*}{$\mathrm{RP}$} & - & - & - & - & - & - & - & - & 0. & 1 & & & & & & & & & & & & \\
\hline & $.83^{*}$ & 0.7 & .7 & 0.8 & 0.69 & 0.7 & 0.45 & 0.6 & 65 & & & & & & & & & & & & & \\
\hline & $*$ & $3^{*}$ & 5* & $0 * *$ & $*$ & $1^{*}$ & & 3 & & & & & & & & & & & & & & \\
\hline \multirow[t]{3}{*}{$\mathrm{TA}$} & - & 0.0 & - & - & - & - & - & - & 0. & 0. & 1 & & & & & & & & & & & \\
\hline & 0.05 & 5 & 0. & 0.3 & 0.66 & 0.6 & 0.29 & 0.3 & 67 & 22 & & & & & & & & & & & & \\
\hline & & & 12 & 9 & & 6 & & 7 & & & & & & & & & & & & & & \\
\hline \multirow[t]{3}{*}{$\mathrm{FI}$} & - & - & - & - & - & - & - & - & 0. & 0. & 0. & 1 & & & & & & & & & & \\
\hline & 0.47 & 0.4 & 0. & 0.6 & 0.69 & 0.6 & 0.03 & 0.4 & 34 & 35 & 40 & & & & & & & & & & & \\
\hline & & 4 & 45 & 2 & $*$ & 2 & & 0 & & & & & & & & & & & & & & \\
\hline
\end{tabular}

decrease at $200 \mathrm{mg} \mathrm{L}^{-1}$. The same was occurred under $100 \mathrm{mg} \mathrm{L}^{-1}$ CuONP which recorded the maximum (0.64 $\mu \mathrm{g}$. $\mathrm{g}^{-1}$ Fresh wt.). However, the reducing power activity was moderate and similar under $50 \mathrm{mg} \mathrm{L}^{-1}$ of both conditions.
These compounds showed approximately same response at nano-conditions ( 25 and $50 \mathrm{mg} . \mathrm{L}^{-1}$ CuONP). Total flavonoid contents increased due to 25 and $50 \mathrm{mg} \mathrm{L}^{-1}$, under both conditions, to the double of control, while the substantial rise was at $100 \mathrm{mg} \mathrm{L}^{-1}$ CuONP. 


\begin{tabular}{|c|c|c|c|c|c|c|c|c|c|c|c|c|c|c|c|c|c|c|c|c|c|c|}
\hline & FW & $\begin{array}{c}\text { D } \\
\text { W }\end{array}$ & $\begin{array}{c}\text { W } \\
\mathbf{C}\end{array}$ & SH & $\begin{array}{l}\mathbf{R} \\
\mathbf{L}\end{array}$ & $\begin{array}{l}\text { Ch } \\
-^{\mathbf{a}}\end{array}$ & $\begin{array}{r}\text { Ch } \\
\text { b b }\end{array}$ & $\begin{array}{c}\mathrm{Ca} \\
\text { ro } \\
\mathbf{t}\end{array}$ & $\mathbf{P h}$ & $\mathbf{R P}$ & $\begin{array}{l}\mathbf{T} \\
\mathbf{A}\end{array}$ & $\mathbf{F I}$ & $\begin{array}{c}\mathrm{Pr} \\
0\end{array}$ & LP & HR & $\begin{array}{l}\mathrm{H}_{2} \\
\mathrm{O}_{2}\end{array}$ & $\begin{array}{c}M \\
C\end{array}$ & $\begin{array}{c}\text { LO } \\
X\end{array}$ & $\begin{array}{c}\text { SO } \\
\text { D }\end{array}$ & $\begin{array}{l}\mathbf{P} \\
\mathbf{O} \\
\mathrm{D} \\
\end{array}$ & CAT & $\begin{array}{l}\mathbf{A} \\
\mathbf{P} \\
\mathbf{X}\end{array}$ \\
\hline $\operatorname{Pr}$ & - & - & - & - & - & - & - & - & 0. & 0.5 & 0. & 0. & 1 & & & & & & & & & \\
\hline \multirow[t]{3}{*}{0} & 0.3 & 0 & 0.1 & 0 & 0. & 0. & 0 & 0. & 62 & 3 & 5 & 3 & & & & & & & & & & \\
\hline & 8 & 17 & 5 & 39 & 5 & 74 & 79 & 77 & & & 2 & 2 & & & & & & & & & & \\
\hline & & & & & 6 & $*$ & $*$ & $*$ & & & & & & & & & & & & & & \\
\hline \multirow[t]{4}{*}{ LP } & 0.8 & 0. & 0.8 & 0. & 0. & 0. & 0. & 0. & - & - & - & - & - & 1 & & & & & & & & \\
\hline & $0^{*}$ & 75 & $9 *$ & 73 & 5 & 56 & 19 & 45 & 0 & 0.9 & 0. & 0. & 0. & & & & & & & & & \\
\hline & $*$ & $*$ & $*$ & $*$ & 9 & & & & 42 & $4^{* *}$ & 0 & 4 & 3 & & & & & & & & & \\
\hline & & & & & & & & & & $*$ & 8 & 3 & 1 & & & & & & & & & \\
\hline $\mathrm{H}$ & 0.7 & 0. & 0.8 & 0. & 0. & 0. & 0. & 0. & - & - & - & - & - & 0.9 & 1 & & & & & & & \\
\hline \multirow[t]{3}{*}{$\mathrm{R}$} & $6^{*}$ & 66 & $0^{*}$ & 73 & 6 & 65 & 36 & 58 & 0 & 0.9 & 0. & 0. & 0. & $6 * *$ & & & & & & & & \\
\hline & & & $*$ & $*$ & 4 & & & & 57 & $8^{* *}$ & 1 & 3 & 4 & $*$ & & & & & & & & \\
\hline & & & & & & & & & & $*$ & 8 & 0 & 1 & & & & & & & & & \\
\hline $\mathrm{H}_{2}$ & 0.6 & 0. & 0.7 & 0. & 0. & 0. & 0. & 0. & - & - & - & - & - & 0.8 & .91 & 1 & & & & & & \\
\hline 0 & 1 & 59 & $4^{*}$ & 61 & 4 & 42 & 15 & 28 & .5 & 0.8 & 0. & 0. & 0 & $7 * *$ & $1^{* *}$ & & & & & & & \\
\hline \multirow[t]{2}{*}{2} & & & & & 4 & & & & 1 & $7^{* *}$ & 1 & 0 & 2 & & $*$ & & & & & & & \\
\hline & & & & & & & & & & & 8 & 5 & 0 & & & & & & & & & \\
\hline$M$ & 0.8 & 0. & 0.7 & 0. & 0. & 0. & 0. & 0. & - & - & - & - & - & 0.8 & .95 & 0.8 & 1 & & & & & \\
\hline \multirow[t]{3}{*}{$C$} & $1^{*}$ & 68 & $8^{*}$ & 73 & 6 & 68 & 50 & 61 & 0 & 0.9 & 0. & 0. & 0 & $9 * *$ & $1^{* *}$ & $8^{*}$ & & & & & & \\
\hline & $*$ & $*$ & & $*$ & 6 & $*$ & & & 57 & $7^{* *}$ & 2 & 2 & 5 & & $*$ & $*$ & & & & & & \\
\hline & & & & & & & & & & $*$ & 5 & 2 & 6 & & & & & & & & & \\
\hline $\mathrm{L}$ & - & 0 & - & - & - & - & - & - & 0 & 0.4 & 0. & - & 0 & - & - & - & - & 1 & & & & \\
\hline 0 & 0.0 & 01 & 0.2 & 0 & 0. & 0. & 0. & 0 & 68 & 5 & 5 & 0. & 1 & 0.3 & 0.5 & 0.6 & 0. & & & & & \\
\hline \multirow[t]{2}{*}{$x$} & 3 & & 0 & 36 & 4 & 41 & 15 & 28 & $*$ & & 5 & 0 & 2 & 5 & 3 & 2 & 42 & & & & & \\
\hline & & & & & 1 & & & & & & & 2 & & & & & & & & & & \\
\hline$S$ & - & - & - & - & - & - & 0. & - & 0 & 0.7 & 0. & 0. & - & - & - & - & - & 0. & 1 & & & \\
\hline 0 & 0.6 & 0 & 0.8 & 0 & 0. & 0 & 15 & 0 & 31 & $7^{*}$ & 0 & 3 & 0 & 0.9 & 0.8 & 0.8 & 0 & 42 & & & & \\
\hline \multirow[t]{2}{*}{$\mathrm{D}$} & 3 & 69 & $3^{*}$ & 63 & 4 & 33 & & 17 & & & 2 & 9 & 0 & $2^{* *}$ & $3^{* *}$ & $5^{*}$ & 70 & & & & & \\
\hline & & $*$ & $*$ & & 3 & & & & & & & & 3 & $*$ & & $*$ & $*$ & & & & & \\
\hline$P$ & - & - & - & - & - & - & 0. & - & 0. & 0.4 & 0. & 0. & - & - & - & - & - & 0. & 0.5 & 1 & & \\
\hline 0 & 0.3 & 0 & 0.4 & 0 & 0. & 0 & 08 & .1 & 42 & 4 & 2 & 1 & 0 & 0.4 & 0.5 & 0.5 & 0 & 71 & 9 & & & \\
\hline \multirow[t]{2}{*}{$D$} & 9 & 41 & 6 & 61 & 5 & 36 & & 3 & & & 4 & 6 & 2 & 5 & 2 & 8 & 40 & $*$ & & & & \\
\hline & & & & & 1 & & & & & & & & 8 & & & & & & & & & \\
\hline$C$ & - & - & - & - & - & - & 0. & 0. & 0. & 0.5 & - & 0. & - & - & - & - & - & 0. & 0.8 & 0. & 1 & \\
\hline$A$ & 0.6 & 0. & 0.8 & 0 & 0. & 0. & 36 & 07 & 01 & 3 & 0. & 4 & 0 & 0.7 & 0.5 & 0.6 & 0 & 16 & $8 *$ & 5 & & \\
\hline \multirow[t]{2}{*}{$\mathrm{T}$} & 1 & 69 & $7^{*}$ & 54 & 3 & 19 & & & & & 0 & 2 & 2 & $5^{*}$ & 9 & 5 & 51 & & $*$ & 5 & & \\
\hline & & $*$ & $*$ & & 7 & & & & & & 1 & & 5 & & & & & & & & & \\
\hline$A$ & - & - & - & - & - & - & 0 & 0 & 0 & 0.5 & - & 0. & - & - & - & - & - & 0 & 0.8 & 0. & 0.9 & 1 \\
\hline$P$ & 0.6 & 0. & 0.7 & 0 & 0. & 0 & 32 & 03 & 04 & 3 & 0. & 4 & 0 & 0.7 & 0.5 & 0.6 & 0. & 14 & $9 *$ & 5 & $7^{* *}$ & \\
\hline \multirow[t]{2}{*}{$x$} & 1 & 75 & $9 *$ & 56 & 3 & 18 & & & & & 0 & 0 & 2 & $4^{*}$ & 8 & 5 & 49 & & $*$ & 3 & $*$ & \\
\hline & & $*$ & & & 5 & & & & & & 6 & & 6 & & & & & & & & & \\
\hline \multirow[t]{4}{*}{$\mathrm{cu}$} & - & - & - & - & - & - & - & - & - & 0.5 & - & 0. & 0 & - & - & - & - & - & 0.3 & 0. & 0.3 & 0. \\
\hline & 0.7 & 0. & 0.5 & 0. & 0. & 0 & 0. & 0. & 0 & 0 & 0. & 1 & 2 & 0.5 & 0.4 & 0.2 & 0 & 0. & 2 & 0 & 4 & 4 \\
\hline & $7^{*}$ & 73 & 2 & 49 & 4 & 41 & 49 & 53 & 02 & & 2 & 7 & 5 & 0 & 5 & 9 & 54 & 31 & & 6 & & 5 \\
\hline & & $*$ & & & 0 & & & & & & 5 & & & & & & & & & & & \\
\hline
\end{tabular}

\section{$* * *=$ Correlation is significant at the 0.001 level \\ $* *=$ Correlation is significant at the 0.01 level \\ * = Correlation is significant at the 0.05 level}

Protective reactions those occurred under toxicity stress to restrict the production of ROS such as hydroxyl radiclescavenging, $\mathrm{H}_{2} \mathrm{O}_{2}$ scavenging, metal chelating and lipid peroxidation inhibition, were represented in table 2. Notably, these four attributes trended similarly and significantly decreased gradually toward the increase in CuO till $150 \mathrm{mg} \mathrm{L}^{-1}$ and return to increase slightly at $200 \mathrm{mg} \mathrm{L}^{-1}$.Also they statistically decreased under CuONP concentrations in comparison to bulk $\mathrm{CuO}$ and control specially at $100 \mathrm{mg} \mathrm{L}^{-1}$, while there were no significant differences between 25 and $50 \mathrm{mg} \mathrm{L}^{-1}$ of this condition. Specifically, $\mathrm{H}_{2} \mathrm{O}_{2}$ radicle scavenging reactions were significantly higher than or equal to the control at 25 and $50 \mathrm{mg} \mathrm{L}^{-1}$ for both bulk and nano-conditions.

The enzymatic antioxidants (SOD, POD, CAT and APX)and lypoxygenase LOX, were almost showed the same direct relationbetween their responseand the increase in $\mathrm{CuO}$ toxicity under bulk (till $150 \mathrm{mg} \mathrm{L}^{-1}$ ) and nano-conditions (till $100 \mathrm{mg} \mathrm{L}^{-1}$ ), while the 200 under bulk-conditions recorded significant decrease even than the control 
(Table 3). This concept can be applied completely on lipoxygenase activity (LOX), meanwhile superoxide dismutase seems to be unaffected at 25 and $50 \mathrm{mg} \mathrm{L}^{-1}$ for both conditions then jumped at $100 \mathrm{mg} \mathrm{L}^{-1}$ CuONP for 8 folds of the control and at $150 \mathrm{mg} \mathrm{L}^{-1} \mathrm{CuO}$ for 4 folds of the control. Also peroxidase, recoded significant increase at $50 \mathrm{mg} \mathrm{L}^{-1}$ than the controlwith the maximum activity at $100 \mathrm{mg} \mathrm{L}^{-1}$ for both conditions. Ascorpate peroxidase and catalase activities at 25 and $50 \mathrm{mg} \mathrm{L}^{-1}$ were more or less equal to those of the control while it was different for $100 \mathrm{mg} \mathrm{L}^{-1}$.

Table 4 show all possible positive and negative correlations among all assessed parameters. Significant positive correlations were found among plant biomass parameters (fresh weight, dry weight, shoot height and root length). Plant biomass production was also positively correlated with different antioxidant ability attributes such as hydroxyl radicle scavenging, inhibition of lipid peroxidation, metal chelating. However, there was negative correlation in plant biomass with the nonenzymatic antioxidants (free phenolics and flavonoids) and Copper contents. The reducing power components showed clear significant positive correlations with all assessed enzymatic antioxidants (SOD, CAT and APX), meanwhile these enzymes were also positively correlated to each other. However, there were significant negative correlations in the relation between these reducing power components on one hand and biomass, leaf pigments and the other antioxidant ability parameters on the other hand.

Subjection of the original data of all measured parameters to the Principle Component Analysis (PCA) interpreted in Figure1 which revealed the previously mentioned correlations on its first two axes. PCA axis 1 captures about $53.3 \%$ of the cumulative percentage followed by the second axis (19.2\%). The distances between the attributes on axis 1 illustrate the degree of similarity; the closer the distance, the greater the resemblance and vice versa. Thus PCA biplot indicated great contrariness between the growth indicators and antioxidant activities (the right hand side of Figure 1) and Copper, enzymatic and non-enzymatic antioxidants contents (the left hand side).

Similarities among different studied attributes represented in the dendrogram (Figure 2) shows that studied attributes can be categorized in four major classes (A-D). Respectively as they shown in the dendrogram; class $A$ included the growth attributes and most of the measured antioxidant ability components. Class B included the leaf pigments (Chlorophyll $a, b$ and carotenoids). Meanwhile, class $C$ included all of the measured enzymatic antioxidants, while $D$ had the soluble proteins with total antioxidants, total phenolic compounds and total flavonoids.

\section{Discussion}

This study was undertaken to investigate the toxicity of CuO and CuONPs in Zea mays seedling development, growth and physiological responses. The toxic effect of engineered nanoparticles in plants used to be studied through various indicatorssuch as germination percentage, biomass production, shoot length, and root growth which were primarily based on studying the effect of heavy metals in plants (OECD, 2004). Based on previous studies, the phytotoxic dose of $\mathrm{CuO}$ nanoparticles varies according to the plant species. For example, Wu et al. (2012) reported that the phytotoxic dose of CuONPs was $397.6 \mathrm{mg} \mathrm{L}^{-1}$ for radish, $175.4 \mathrm{mg} \mathrm{L}^{-1}$ for cucumber and $12.9 \mathrm{mg} \mathrm{L}^{-1}$ for lettuce. In the present study, the phytotoxic concentrations of CuONPs those inhibited the seeds germinations at all were those equal or smaller than $150 \mathrm{mg} \mathrm{L}^{-1}$ while they can withstand till $100 \mathrm{mg} \mathrm{L}^{-1}$.

Shi et al. (2014) in a study on Elshotzia splendens concluded that the phytotoxicity was due to CuONPs exposure and not from soluble $\mathrm{Cu}$. In an earlier study, Lee et al. (2008) have also reported that exposure to very low concentrations of $\mathrm{Cu}^{2+}$ ions has not affected the shoot and root growth in Phaseolus radiates and Triticum aestivum. Also Prakash et al. (2014) recorded drastic roots-growth retardation and triggering their lignification in Soybeanunder 400-500 $\mathrm{mg} \mathrm{L}^{-1}$ CuONPs. In accordance with the present study, the exposure to the highest

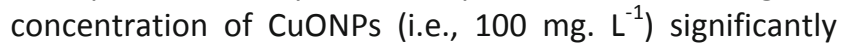
reduced the shoot and root growth as well as the total chlorophyll and carotenoids contents in maize seedlings. Hence, Chlorophyll is the critical photosynthetic pigment and its levels can be a significant indicator of toxicity to plants (Ma et al., 2015). However, exposure to CuO made the same effect at double of this dose $\left(200 \mathrm{mg}^{-1}\right)$. This might be due to the fact that lower concentration of $\mathrm{Cu}$ is essential for plant development since it is an essential micronutrient for the plant growth. However, higher exposure concentrations of CuO-nanoparticles might have resulted in the excess presence of $\mathrm{Cu}$ in maize roots leading to adverse effects on plant development (Prakash et al., 2014).

A very high concentration of nanoparticles may severely affect the photosynthesis which may result in plant growth suppression or plant death. Several reports have observed significant decrease in plant growth and pigments as the result of nanoparticle exposure as in barely (Shaw et al., 2014) and rice (Costa and Sharma, 2016). The significant reduction in chlorophyll attributes, as observed in this study, might be due to the reduction in biomass or as a result of the lipid peroxidation of chloroplast membranes due to the NP oxidative stress (Ma et al., 2013). Similarly, significant reduction in chlorophyll attributes as a result of AgNPs exposure had also been reported by Ma et al. (2013) from Arabidopsis thaliana. While, unusual increase in Chlorophyll $b$ and carotenoids at $100 \mathrm{mg} \mathrm{L}^{-1}$ CuONPwas reported with tomato under $250 \mathrm{mg} \mathrm{TiO}_{2} \mathrm{NP} \mathrm{Kg}^{-1}$ (Raliya et al., 2015) and $500 \mathrm{mg} \mathrm{Ce}_{2} \mathrm{ONP} \mathrm{Kg}{ }^{-1}$ soil (Barrios et al., 2016).

Previous studies reported that the surface of NPs could be covered by various macromolecules, and the chemical surface property and the ligand density of NPs could strongly influence the interaction between NP-bound ligands and cellular receptors (Rauch et al., 
2013). This could be due to the fact that macromolecules in root exudates altered physicochemical properties of the NPs surface and resulted in NPs accumulation in the root epidermis (Ghafariyan et al., 2013). The plants grown in presence of nanoparticles may absorb and translocate them in different tissues. It has been shown that $\mathrm{CuO}$ nanoparticles were reduced to $\mathrm{Cu}_{2} \mathrm{O}$ and $\mathrm{Cu}_{2} \mathrm{~S}$ in maize plants (Wang et al., 2012). Several studies in Zea mays seedlings have proved that the uptake and translocation of CuONPs occurs through the roots to shoots via xylem and shoots to roots via phloem (Wang et al., 2012). Moreover, Shi et al. (2014) reported that once inside the plant cells, the dissolution of CuONPs are promoted due to the reduced $\mathrm{pH}$ and by their interaction with organic acids and proteins inside the plant tissues. In accordance with earlier reports, $\mathrm{Cu}$ metal content analysis provided evidence for the presence of significantly high Cu content in roots of CuONP-exposed soybean seedlings (Prakash et al., 2014) and our maize seedlings.

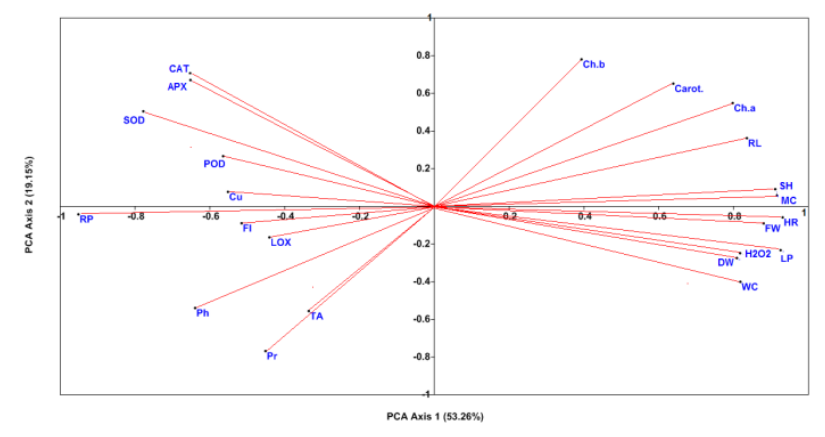

Figure 1. Loading plot of different studied attributes correlations to the first two Principle component analysis (PCA) axes.

Horizontal and vertical arrows indicate the rise-direction of $\mathrm{CuO}$ and CuONP concentrations. Abbreviations: $\mathrm{Cu}=$ Cupper, $\mathrm{Ph}=$

Total Phenolics, $\mathrm{TA}=$ Total Antioxidants, $\mathrm{Pr}=$ Proteins, $\mathrm{FI}=$ Total

Flavonoids, RP = Reducing Power, $\mathrm{LOX}=$ Lipoxygenase, $\mathrm{POD}=$

Peroxidase, $\mathrm{SOD}=$ Superoxide Dismutase, $\mathrm{CAT}=$ Catalase, $\mathrm{APX}=$ Ascorbate peroxidase, $\mathrm{Ch} . \mathrm{A}=$ Chlorophyll $\mathrm{a}, \mathrm{Ch} . \mathrm{b}=$

Chlorophyll b, Carot. $=$ Carotenoids, $\mathrm{LP}=$ Lipid peroxidase, $\mathrm{HR}=$

Hydroxyl reductase, $\mathrm{MC}=$ Metal chelating agents, $\mathrm{H}_{2} \mathrm{O}_{2}=$

Hydrogen peroxide scavenging, $\mathrm{WC}=$ Water content, $\mathrm{RL}=$ Root length, $\mathrm{SH}=$ Shoot height, $\mathrm{DW}=$ Dry weight, $\mathrm{FW}=$ Fresh weight.

While redox biology implies a slight increment of the reactive oxygenated species level, meant to activate signaling pathways, oxidative stress involves elevated ROS amounts, resulting in the impairment of biomolecules such as nucleic acids, protein or lipids (Schieber and Chandel, 2014).

Oxidative stress was characterized by Sies (1991) as "a disturbance in the prooxidant to antioxidant balance in favor of the oxidant species, leading to potential damage". Oxidative stress has been understood as an excessive amount of ROS that is the outcome of an imbalance between the generation and depletion of ROS. Hence, oxidative stress is the repercussion of an enhanced free radical occurrence, but also of a reduced activity of the protective antioxidant defense system (Poljsak et al., 2013).

From this point of view, the present study followed the incidence of oxidative stress resulted from $\mathrm{Cu}$ toxicity (CuO and CuONP) through detecting the activity of the protective antioxidant defense system. The general evaluation took place by detecting total antioxidant potentialandreducing power. While more specific evaluation performed through detecting some of the enzymatic antioxidants (SOD, CAT, APX, and POD) and non-enzymatic antioxidants (phenolics, flavonoids, carotenoids). The outcome of the protective antioxidant defense system in depleting ROS evaluated through conducting the change in lipid peroxidation inhibition\%, $\mathrm{OH} \cdot \& \mathrm{H}_{2} \mathrm{O}_{2}$ free radicals scavenging\%, and metal chelating\%.

The phosphomolybdenum assay has been routinely used to evaluate the totalantioxidant potential of extracts (Prieto et al., 1999). In the presence of antioxidants, Mo (VI) is reduced to Mo (V) and forms a green colored phospho-molybdenum $\mathrm{V}$ complex. The data showed $\mathrm{a}$ clear and a significant induction in total antioxidant potential as consequence of $\mathrm{Cu}$ toxicity ( $\mathrm{CuO}$ and $\mathrm{CuONP}$ ) which was more obvious under Cuo when compared to CuONP.On the other hand, the measurement of reducing power of the maize cells under $\mathrm{Cu}$ toxicity may serve as a significant indicator of its potential antioxidant activity. Our data showed that excess $\mathrm{Cu}$ (micro and nano) evacuated the reducing power of the cells as compared to control. But unlike total antioxidants data, reducing power was higher under CuONP $\left(25,50,100 \mathrm{mg} \mathrm{L} \mathrm{L}^{-1}\right)$ in comparison to $\mathrm{CuO}$ bulk. This could be attributed to the difference in sensitivity between the two assays.

Statistical evaluation bypearsoncorrelation between the TAC assay and reducing power was found to be non-significant $(r=0.22)$. On the other hand, correlation between TAC and total phenolic contents was found to be significant $(r=0.67)$. Another significant correlation was observed between FRAP assay and the total phenolic contents $(r=0.65)$. This indicates that phenolic compounds might be a major contributor to the antioxidant capacities under $\mathrm{Cu}$ toxicity. A high correlation between the total phenolic content and antioxidant activity has been reported by many researchers (Chew et al., 2008; Wang et al., 2009). Lipids are the most susceptible biomolecules to undergo oxidation: polyunsaturated fatty acids which lead to malondialdehyde, a recognized marker of lipid oxidative decay and their levels are considered to accurately reflect the oxidative stress (Pisoschi and Pop, 2015).

Increased LOX activity, presented in this work under $\mathrm{Cu}$ bulk as compared to CuNPat ( 25 and $100 \mathrm{mg} \mathrm{L}^{-1}$ ) treatments suggests higher lipolytic activity of the membranes and oxidation of membrane-bound fatty acids, which propagates higher lipid peroxidation (Tavallali et al., 2010). Surprisingly, the data of lipid peroxidation inhibition\%, shows higher level of membranes protection 
under $\mathrm{Cu}$ bulk when compared to corresponding CuNP concentrations ( $\left.25 \& 100 \mathrm{mg} \mathrm{L}^{-1}\right)$. This could be attributed to the non-enzymatic biosynthesis of lipid peroxides. Oxidized lipids in addition to being synthesized in a specific and controlled manner by lipoxygenase (LOX), the non-enzymatic peroxidation of lipids could be mediated by carbon- and oxygen-centered radicals. Like all radical reactions, this process can be broken down into three discrete phases: initiation, propagation, and termination (Yousri et al., 2011). Yet, the substrate scope and general mechanism of lipid peroxidation is largely the same in both cases.

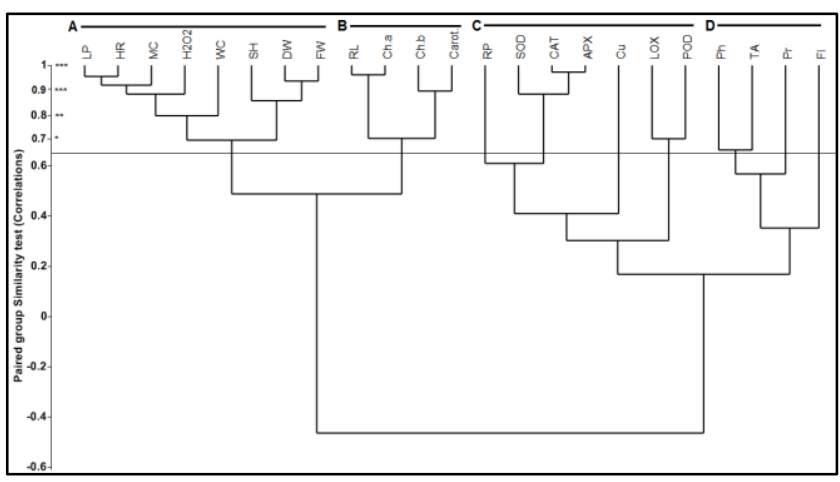

Figure 2. Cluster analysis of measured attributes show significance, ${ }^{*}$ at $p \leq 0.05,{ }^{* *}$ at $p \leq 0.01$ and ${ }^{* * *}$ at $p \leq 0.001$. Letters (A-D) refer to the clusters of similar trends

Nanoparticles may interfere with plant metabolism in several ways, such as by providing micronutrients (Liu and Lal, 2015), regulation of genes (Nair and Chung, 2014), or interfering with different oxidative processes in plants which results in oxidative burst (Hossain et al., 2015). Some studies have demonstrated that nanoparticle exposure improves free-radical scavenging potential and antioxidant enzymatic activities and alters microRNAs expression that regulates different morphological, physiological and metabolic processes in plants (Siddiqi and Husen, 2016b). It is clear that several nanoparticles when present in excess results into ROS production, and interfere with the oxidative mechanism (Anshu et al., 2017).

Redox homeostasis of the cell is ensured by its complex endogenous antioxidant defense system. Attempts were made to classify antioxidant systems from the reactivity standpoint: the so called "first line of defense" has been identified as the enzymatic antioxidant system, including superoxide dismutase which depletes superoxide radical anion $\mathrm{O}_{2}$, catalase and peroxidase, and also the ascorbate peroxidase that decomposes $\mathrm{H}_{2} \mathrm{O}_{2}$. The "second line of defense" is represented mainly by reduced thiols and low molecular-weight antioxidants. The latter include a broad range of molecules, both hydro- and liposoluble (tocopherols, ascorbate, polyphenols, etc.) or metabolic compounds (ascorbate and reduced glutathione), and low molecular weight scavengers, like lipoic acid (Poljsak et al., 2013). These compounds impart basically the antioxidant capacity to biological media. The respective biomolecules can reach particular locations in cells affected by the oxidative attack (Tessutti et al., 2013).
All measured enzymatic antioxidants (SOD, CAT, APX and POD) activities were increased in the plumules of Zea mays gradually with increase in bulk-CuO concentration to the double of control values at $150 \mathrm{mg}$. $\mathrm{L}^{-1}$. Meanwhile, this rise was faster, at 25 and $50 \mathrm{mg} \mathrm{L}^{-1}$, under nanoconditions. It has been already reported that these antioxidant enzymes can protect plant cells against the adverse effects of reactive oxygen species (Das and Roychoudhury, 2014). The increase of CAT activity in leaves under NPs stress suggested that its effective scavenging mechanism to remove $\mathrm{H}_{2} \mathrm{O}_{2}$ resulted from metal stress caused oxidative damage (Reddy et al., 2005). While POD acts as scavenger of ROS, SOD and CAT jointly convert $\mathrm{O}_{2}^{-}$and $\mathrm{H}_{2} \mathrm{O}_{2}$ to $\mathrm{H}_{2} \mathrm{O}$ and $\mathrm{O}_{2}$ and also reduce overall free $\mathrm{OH}$. radical which suggested that this enzyme served as an intrinsic defense tool to resist $\mathrm{CuO}$ and CuONP induced oxidative damage in maize plants. This confirms the regulation of antioxidant system as a response to nanoparticle interaction with maize plants. It has been reported that excess $\mathrm{Cu}$ triggers the generation of ROS and free radicals and thus causes molecular damage to plants (Liu et al., 2004). Reactive oxygen species (ROS) are represented by both free radical and non-free radical oxygenated molecules such as hydrogen peroxide $\left(\mathrm{H}_{2} \mathrm{O}_{2}\right)$, superoxide $\left(\mathrm{O}_{2}^{-}\right)$, singlet oxygen $\left(1 / 2 \mathrm{O}_{2}\right)$, and the hydroxyl radical $(\mathrm{OH} \cdot)$. Reactive nitrogen, iron, copper, and sulfur species are also encountered (Riley, 1994).

Following the outcome of the protective antioxidant defense system $\left(\mathrm{OH} \cdot \& \mathrm{H}_{2} \mathrm{O}_{2}\right.$ free radicals scavenging \%, and metal chelating \%), the data obtained proved the increased antioxidants' ability of maize cells under bulk $\mathrm{Cu}$ toxicity as compared to Nano $\mathrm{Cu}$ toxicity to contain the effects of reactive oxygen species activity, and delay the incidence of cell death obviously at $\left(25 \& 100 \mathrm{mg} \mathrm{L}^{-1}\right)$. This implies higher oxidative stress incidence under CuNPdespite of higher enzymatic antioxidants (SOD, CAT, POD and APX) and non-enzymatic antioxidants (phenolics, flavonoids). If the antioxidant produced are unable to control the ROS, the ROS oxidized the cell macromolecules (Sharma et al., 2012), which ultimately results in the death of the plant.

The concept of biological antioxidant refers to any compound that, when present at a lower concentration compared to that of an oxidizable substrate, is able to either delay or prevent the oxidation of the substrate (Godic et al., 2014). Antioxidant functions imply lowering oxidative stress, as well as other parameters of cell damage.

It's apparent from the data obtained herein, that both first and second line of defense could not help the plant to survive the elevating concentrations of CuNP. Nevertheless, it could be proposed that mainly at sustained free radical action, the defense system's capacity against ROS can be overwhelmed, leading to retarded growth and death occurrence. Hence, Seed germination was completely stopped under 150 and $200 \mathrm{mg} \mathrm{L}^{-1}$ CuONP. These concentrations showed the same or more drastic effect than 150 and $200 \mathrm{Cu}$ bulk, and 
showed that Nano sized particles of $\mathrm{CuO}$ proved more phytotoxicity than bulk particles.

Taking over these considerations, it is important to remind that the novel concept of oxidative stress is not restricted to free radical damage of the biomolecules, but relies on identifying perturbation of cellular redox status (LopezAlarcon and Denicola, 2013). Based on many studies on redox signaling pathways, on antioxidant mechanism and oxidative stress markers, Dean Jones re-defines oxidative stress as "a disruption in redox signaling and control", hence the action of the antioxidant systems is viewed as more complicated than merely blocking reactive free radicals (Jones, 2006). Higher activity of antioxidants (enzymatic and non-enzymatic) obtained herein, which accompanied with higher reducing power content could lead to more disruption in the cell redox signaling rather than blocking ROS, this disruption may not help the cell to survive under $\mathrm{Cu}$ toxicity stress, particularly at germination and plant establishment stage which is comprehensively regulated by multiple factors, and the presence of reactive oxygen species (ROS) at particular levels are among these factors (Schopfer et al., 2001). A confirmation of this assumption could be gathered from the analysis of Principle component (PCA), that showed a reduction in growth parameters with the rise in CuONP concentrations, which in turn accompanied with increment in the activity of almost all antioxidants and reducing power.

It can be concluded that seedlingsgrowth, development and pigments followed the same trend and they were can not cope with CuOand CuONP phytotoxicity. Nano sized particles of $\mathrm{CuO}$ proved more phytotoxicity than bulk particles and causedcomplete germination retardation under 150 and $200 \mathrm{mg} \mathrm{L}^{-1}$ CuONP. These concentrations showed the same or more drastic effect than 150 and 200 $\mathrm{Cu}$ bulk. Higher enzymatic antioxidants (SOD, CAT, POD and $\mathrm{APX}$ ) and non-enzymatic antioxidants (phenolics and flavonoids) could not sustain the survival of maize seedlings under elevating concentrations of CuNP. The capacity of defense systems against ROS could be overwhelmed at sustained free radical action, leading to growth retardation and death incidence.

\section{References}

Aebi H. (1984), Catalase. In Methods in Enzymology, Paker I. (ed.) Vol. 105, Academic press, Orlando, Fl., USA.

Anshu R., Marek Z., Oksana S., Hazem M., Kalaji X.H, Mbarki S. and Brestic M. (2017), Impact of metal and metal oxide nanoparticles on plant: A critical review, Frontiers in Chemistry, 5, 1-16.

Atha D.H., Wang H., Petersen E.J., Cleveland D., Holbrook R.D., Jaruga P., Dizdaroglu M., Xing B. and Nelson B.C. (2012), Copper oxide nanoparticle mediated DNA damage in terrestrial plant models, Environmental Science \& Technology, 46, 1819-1827.

AzevedoNeto A.D., Gomes-Filho E. and Prisco J.T. (2008), Salinity and oxidative stress. In: Khan N.A. and Sarvajeet S. (Eds.), Abiotic Stress and Plant Responses, IK International, New Delhi.
Barbara D.W. (1990), Role of ferritin as a lipid oxidation catalyst in muscle food, Journal of Agricultural and Food Chemistry, 38, 674-677.

Barrena R., Casals E., Colon J., Font X., Sanchez A., Puntes V. (2009), Evaluation of the ecotoxicity of model nanoparticles. Chemosphere, 75, 850-857.

Barrios A.C., Rico C.M., Trujillo-Reyes J., Medina-Velo I.A., Peralta-Videa J.R. and Gardea-Torresdey J.L. (2016), Effects of uncoated and citric acid coated cerium oxide nanoparticles, bulk cerium oxide, cerium acetate, and citric acid on tomato plants. Science of the Total Environment, 563, 956-964.

Chew Y.L., Lim Y.Y., Omar M. and Khoo K.S. (2008), Antioxidant activity of three edible seaweeds from two areas in South East Asia, Learning with Technologies, 41, 1067-1072.

Costa M.V.J.D. and Sharma P.K. (2016), Effect of copper oxide nanoparticles on growth, morphology, photosynthesis, and antioxidant response in Oryza sativa, Photosynthetica, 54, 110-119.

Das K. and Roychoudhury A. (2014), Reactive oxygen species (ROS) and response of antioxidants as ROS-scavengers during environmental stress in plants, Frontiers of Environmental Science, 2, 53.

Dimkpa C., Merten D., Svatoš A., Büchel G. and Kothe E. (2008), Hydroxamatesiderophores produced by Streptomyces acidiscabies E13 bind nickel and promote growth in cowpea (Vignaunguiculata L.) under nickel stress, Canadian Journal of Microbiology, 54, 163-172.

Dimkpa C.O., Calder C., Gajjar P., Merugu S., Huang W., Britt D.W., McLean J.E., Johnson W.P. and Anderson A.J. (2011), Interaction of silver nanoparticles with an environmentally beneficial bacterium, Pseudomonas chlororaphis, Journal of Hazardous Materials, 188, 428-435.

Dimkpa C.O., McLean J.E., Britt D.W. and Anderson A.J. (2012), $\mathrm{CuO}$ and $\mathrm{ZnO}$ nanoparticles differently affect the secretion of fluorescent siderophores in the beneficial root colonizer, Pseudomonas chlororaphis 06, Nanotoxicology, 6, 635-642.

Du W., Sun Y., Ji R., Zhu J., Wu J. and Guo H. (2011), $\mathrm{TiO}_{2}$ and $\mathrm{ZnO}$ nanoparticles negatively affect wheat growth and soil enzyme activities in agricultural soil, Journal of Environmental Monitoring, 13, 822-828.

Du W., Tan W., Peralta-Videa J.R., Gardea-Torresdey J.L., Ji R., Yin Y. and Guo H. (2017), Interaction of metal oxide nanoparticles with higher terrestrial plants: physiological and biochemical aspects, Plant Physiology and Biochemistry, 110, 210-225.

El-Temsah Y.S. and Joner E.J. (2012), Impact of $\mathrm{Fe}$ and $\mathrm{Ag}$ nanoparticles on seed germination and differences in bioavailability during exposure in aqueous suspension and soil, Environmental Toxicology, 27, 42-49.

Fedovich B.B.C. (1976), Superoxide dismutase assay and an assay applicable to acrylamide gel. Analytical Biochemistry, 10, 276-287.

Fernandes J.C. and Henriques F.S. (1991), Biochemical, physiological and structural effects of excess copper in plants, Botanical Review, 57, 246-273.

Gao F., Liu C., Qu C., Zheng L., Yang F., Su M. and Hong F. (2008), Was improvement of spinach growth by nano- $\mathrm{TiO}_{2}$ treatment related to the changes of rubiscoactivase?, Biometals, 21, 211-217. 
Ghafariyan M., Malakouti M., Dadpour M., Stroeve P. and Mahmoudi M. (2013), Effects of magnetite nanoparticles on soybean chlorophyll, Environmental Science \& Technology, 47, 10645-10652.

Godic A., Poljsak B., Adamic M. and Dahmane R. (2014), The role of antioxidants in skin cancer prevention and treatment, Oxidative Medicine and Cellular Longevity. Article ID 860479, 6 pages, http://dx.doi.org/10.1155/2014/860479.

Halliwell B. and Gutteridge J.M.C. (2007), Free Radicals in Biology and Medicine. Oxford University Press, New York.

Hammer O., Harper D.A.T. and Ryan P.D. (2001), PAST: Paleontological Statistics Software Package for Education and Data Analysis, Palaeontologia Electronica.

Hatami M. and Ghorbanpour M. (2014), Defense enzyme activities and biochemical variations of Pelargonium zonalein response to nanosilver application and dark storage, Turkish Journal of Biology, 38, 130-139.

Hossain Z., Mustafa G. and Komatsu S. (2015), Plant responses to nanoparticle stress, International Journal of Molecular Sciences, 16, 26644-26653.

Janero D.R. (1990), Malondialdehyde and thiobarbituric acid reactivity as diagnostic indices of lipid peoxidation and peroxidative tissue injury, Free Radical Biology and Medicine, 9, 513-540.

Jiang M. and Zhang J. (2002), Water stress-induced abscisic acid accumulation triggers the increased generation of reactive oxygen species and up-regulates the activities of antioxidant enzymes in maize leaves, Journal of Experimental Botany, 53, 2401-2410.

Jones D.P. (2006), Redefining oxidative stress, Antioxidants \& Redox Signaling, 8, 1865-1879.

Kahru A. and Ivask A. (2013), Mapping the dawn of nanoecotoxicological research, Accounts of Chemical Research, 46(3), 823-833.

Keller A.A. and Lazareva A. (2013), Predicted releases of engineered nanomaterials: from global to regional to local, Environmental Science \& Technology Letters, 1, 65-70.

Keller A.A., McFerran S., Lazareva A. and Suh S. (2013), Global life cycle releases of engineered nanomaterials, Journal of Nanoparticle Research, 15, 1692.

Kunchandy M.N.A.R. (1990), Oxygen radical scavenging activity of curcumin, International Journal of Pharmacognosy, 58, 237-240.

Lee W.M., An Y.J., Yoon H. and Kweon H.S. (2008), Toxicity and bioavailability of copper nanoparticles to the terrestrial plants mung bean (Phaseolusradiatus) and wheat (Triticumaestivum): plant agar test for water-insoluble nanoparticles, Environmental Toxicology and Chemistry, 27, 1915-1921.

Lichtenthaler H.K. (1987), Chlorophylls and Carotenoids: Pigments of Photosynthetic Biomembranes, In: Methods in Enzymology, Academic press, Orlando, Fl., USA, vol. 148, pp. 350-382.

Lin D.H, Xing B.S. (2008), Root uptake and phytotoxicity of $\mathrm{ZnO}$ nanoparticles, Environmental Science \& Technology, 42, 5580-5585.

Liu J., Xiong Z., Li T., Huang H. (2004), Bioaccumulation and ecophysiological responses to copper stress in two populations of Rumexdentatus $L$. from Cu contaminated and non-contaminated sites, Environmental and Experimental Botany, 52(1), 43-51.
Liu R. and Lal R. (2015), Potentials of engineered nanoparticles as fertilizers for increasing agronomic productions, Science of the Total Environment, 514, 131-139.

Long L.H., Evans P.J. and Halliwell B. (1999), Hydrogen peroxide in human urine: implications for antioxidant defense and redox regulation, Biochemical and Biophysical Research Communications, 262, 605-609.

Lopez-Alarcona C. and Denicola A. (2013), Evaluating the antioxidant capacity of naturalproducts: a review on chemical and cellular-based assays, Analytica Chimica Acta, 763, 1-10.

Lowry O.H., Rosebrough N.J., Farr A.L. and Randall R.J. (1951), Protein measurement with Folin phenol reagent, Journal of Biological Chemistry, 193, 265-275.

Lu C.M., Zhang C.Y., Wen J.Q. and Wu G.R. (2002), Research on the effect of nanometer materials on germination and growth enhancement of Glycine max and its mechanism, Soybean Science, 21, 68-171.

Ma C., Chhikara S., Minocha R., Long S., Musante C. and White J.C., et al. (2015), Reduced silver nanoparticle phytotoxicity in crambeabyssinica with enhanced glutathione production by overexpressing bacterial g-glutamylcysteine synthase, Environmental Science \& Technology, 49(16), 10117.

Ma C., Chhikara S., Xing B., Musante C., White J. and Dhankher O. (2013), Physiological and molecular response of Arabidopsis thaliana (L.) to nanoparticle cerium and indium oxide exposure, ACS Sustain, Chemical Engineering, 1, 768778.

Minguez-Mosquera M.I., Jaren-Galan M. and Garrido-Fernandez J. (1993), Lipoxygenase activity during pepper ripening and processing of paprika, Phytochemistry, 32, 11031108.

Mohammadi R., Maali-Amiri R. and Abbasi A. (2013), Effect of $\mathrm{TiO}_{2}$ nanoparticles on chickpea response to cold stress, Biological Trace Element Research, 152, 403-410.

Moreno M.I.N., Isla M.I., Sampietro A.R. and Vattuone M.A. (2000), Comparison of the free radical scavenging activity of propolis from several regions of Argentina, Journal of Ethnopharmacology, 71, 109-114.

Nair P.M.G. and Chung I.M (2014), Impact of copper oxide nanoparticles exposure on Arabidopsis thaliana growth, root system development, root lignification, and molecular level changes, Environmental Science and Pollution Research, 21, 12709-12722.

Nair P.M.G. and Chung I.M. (2015), Study on the correlation between copper oxide nanoparticles induced growth suppression and enhanced lignification in Indian mustard (Brassica juncea L.), Ecotoxicology and Environmental Safety, 113, 302-313.

Nair P.M.G., Kim S.H. and Chung I.M. (2014), Copper oxide nanoparticle toxicity in mung bean (Vignaradiata L.) seedlings: physiological and molecular level responses of in vitro grown plants, Acta Physiologiae Plantarum, 36, 29472958.

Nair R., Varghese S.H., Nair N.G., Maekawa T., Yoshida Y. and Kumar D.S (2010), Nanoparticulate material delivery to plants, Plant Science, 179, 154-163.

Navarro E., Baun A., Behra R., Hartmann N.B, Filser J., Miao A.J., Quigg A., Santschi P.H. and Sigg L. (2008), Environmental behavior and ecotoxicity of engineered nanoparticles to algae, plants, and fungi, Ecotoxicology, 17, 372-386. 
OECD (Organization for Economic Cooperation and Development) (2004), Proposal for updating guideline 208. Terrestrial plant test: seedling emergence and seedling growth test, Draft document.

Oyaizu M. (1986), Studies on product of browning effect reaction prepared from glucose amine, Journal of Nutrition, 44, 307315.

Peralta-Videa J.R., Huang Y., Parsons J.G., Zhao L., Lopez-Moreno M.L., HernandezViezcas J.A. and Gardea-Torresdey J.L. (2016), Is the green synthesis of engineered nanomaterials a realistic alternative to chemical synthesis? A review of the factors affecting their mass production and applications, Nanotechnology for Environmental Engineering, 1(1), 4.

Perreault F., Samadani M. and Dewez D. (2014), Effect of soluble copper released from copper oxide nanoparticles solubilisation on growth and photosynthetic processes of Lemnagibba. L. Nanotoxicology, 8, 374-382.

Piccinno F., Gottschalk F., Seeger S. and Nowack B. (2012), Industrial production quantities and uses of ten engineered nanomaterials for Europe and the world, Journal of Nanoparticle Research, 14, 1109-1120.

Pisoschi A.M. and Pop A. (2015), The role of antioxidants in the chemistry of oxidative stress: A review, European Journal of Medicinal Chemistry, 97, 55-74.

Poljsak B., Suput D. and Milisav I. (2013), Achieving the balance between ROS and antioxidants: when to use the synthetic antioxidants, Oxidative Medicine and Cellular Longevity. Article ID 956792, 11 pages.

Prakash M. and Gopalakrishnan Nair III M.C. (2014), A Mechanistic Study on the Toxic Effect of Copper Oxide Nanoparticles in Soybean (Glycine max L.) Root Development and Lignification of Root Cells, Biological Trace Element Research.

Prieto P., Pineda M. and Aguilar M. (1999), Spectrophotometric quantitation of antioxidant capacity through the formation of a phosphomolybdenum complex: specific application to the determination of vitamin E, Analytical Biochemistry, 269, 337-341.

Priyadarshini S., Deepesh B., Zaidi M.G.H., PardhaSaradhi P., Khanna P.K. and Arora S. (2012), Silver nanoparticlemediated enhancement in growth and antioxidant status of Brassica juncea, Applied Biochemistry and Biotechnology, 167, 2225-2233.

Raliya R., Nair R., Chavalmane S., Wang W.N. and Biswas P. (2015), Mechanistic evaluation of translocation and physiological impact of titanium dioxide and zinc oxide nanoparticles on the tomato (Solanumlycopersicum L.) plant. Metallomics, 7, 1584-1594.

Rauch J., Kolch W. and Laurent S. (2013), Mahmoudi M. Big signals from small particles: regulation of cell signaling pathways by nanoparticles, Chemical Reviews, 113(5), 3391-3406.

Reddy A.M., Kumar S.G., Jyothsnakumari J., Timmanaik S., andSudhakar C. (2005), Lead induced changes in antioxidant metabolism of horsegram (Macrotylomauniflorum (Lam.) Verdc.) and bengalgram (Cicerarietinum L.), Chemosphere, 60, 97-104.

Rico C.M., Hong J., Morales M.I., Zhao L., Barrios A.C., Zhang J.Y., Peralta-Videa J.R. and Gardea-Torresdey J.L. (2013), Effect of cerium oxide nanoparticles on rice: a study involving the antioxidant defense system and in vivo fluorescence imaging, Environmental Science \& Technology, 47, 56355642.

Riley P.A. (1994), Free radicals in biology: oxidative stress and the effects of ionizing radiation, International Journal of Radiation Biology, 65, 27-33.

Sawhney B.L. and Frink C.R. (1991), Heavy metals and their leachability in incinerator ash, Water, Air and Soil Pollution, 57-58, 289-296.

Schieber M. and Chandel S.N. (2014), ROS function in redox signaling and review oxidative stress, Current Biology, 24, 453-462.

Schopfer P., Plachy C. and Frahry G. (2001), Release of reactive oxygen intermediates (superoxide radicals, hydrogen peroxide, and hydroxyl radicals) and peroxidase in germinating radish seeds controlled by light, gibberellin, and abscisic acid, Plant Physiology, 125, 1591-1602.

Schrurs F. and Lison D. (2012), Focusing the research efforts, Nature Nanotechnology, 7, 546-548.

Scrinis G. and Lyons K. (2007), the emerging nano-corporate paradigm: nanotechnology and the transformation of nature, food and agri-food systems, International Journal of Sociology of Agriculture and Food, 15, 22-44.

Servin A., Elmer W., Mukherjee A., De La Torre-Roche R., Hamdi H., White J.C. and Dimkpa C. (2015), Nanoscale Micronutrients Suppress Disease, VFRC Report Virtual Fertilizer Research Center, Washington, D.C., p. 33.

Shaw A.K., Ghosh S., Kalaji H.M., Bosa K., Brestic M., Zivcak M. and Hossain Z. (2014), Nano-CuO stress induced modulation of antioxidative defense and photosynthetic performance of Syrian barley (Hordeumvulgare L.), Environmental and Experimental Botany, 102, 37-47.

Shi J., Peng C., Yang Y., Yang J., Zhang H., Yuan X., Chen Y. and Hu T. (2014), Phytotoxicity and accumulation of copper oxide nanoparticles to the Cu-tolerant plant Elsholtziasplendens, Nanotoxicology, 8, 179-188.

Siddiqi K.S. and Husen A. (2016a), Fabrication of metal and metal oxide nanoparticles by algae and their toxic effects, Nanoscale Research Letters, 11, 363.

Siddiqi K.S. and Husen A. (2016b), Engineered gold nanoparticles and plant adaptation potential, Nanoscale Research Letters, 11, 400.

Siddiqi K.S. and Husen A. (2017), Plant Response to Engineered Metal Oxide Nanoparticles, Nanoscale Research Letters, 12, 92.

Sies H. (1991), Oxidative stress: from basic research to clinical application, American Journal of Medicine, 91, 31-38,

Singleton V.L. and Rossi J.A. (1965), Colorimetry of total phenolics with phosphomolyb dicphospho tungstic acid reagents, American Journal of Enology and Viticulture, 16, 144-158.

Tatiana Z., Yamashita K. and Matsumoto H. (1999), Iron deficiency induced changes in ascorbate content and enzyme activities related to ascorbate metabolism in cucumber roots, Plant and Cell Physiology, 40, 273-280.

Tavallali V., Rahemi M., Eshghi S., Kholdebarin B. and Ramezanian A. (2010), Zinc alleviates salt stress and increases antioxidant enzyme activity in the leaves of pistachio (Pistaciavera L. 'Badami') seedlings, Turkish Journal of Agriculture and Forestry, 3, 349-359. 
Tessutti L.S., Macedo D.V., Kubota L.T. and Alves A.A. (2013), Measuring the antioxidant capacity of blood plasma using potentiometry, Analytical Biochemistry, 441, 109-114.

Wang Z., Xie X., Zhao J., Liu X., Feng W., White J.C. and Xing B. (2012), Xylem- and Phloem-based transport of CuO nanoparticles in maize (Zea mays L.), Environmental Science \& Technology, 46, 4434-4441.

Wang T., Jonsdottir R. and Ólafsdóttir G. (2009), Total phenolic compounds, radical scavenging and metal chelation of extracts from Icelandic seaweeds, Food Chemistry, 116(1), 240-248.

Wu S.G., Huang L., Head J., Chen D.R., Kong I.C. and Tang Y.J. (2012), Phytotoxicity of metal oxide nanoparticles is related to both dissolved metals ions and adsorption of particles on seed surfaces, Journal of Petroleum and Environmental Biotechnology, 3, 1000126.

Yousri R., Noaman E., El Shawi O., Fahmy N. and Ghaz M. (2011), Evaluation of antioxidant status and radioprotective activity of a novel anti-cancer drug in mice, Journal of Cancer Therapy, 2, 616-628.

Zhao L., Peng B., Hernandez-Viezcas J.A., Rico C., Sun Y., PeraltaVidea J.R., Tang X., Niu G., Jin L., Varela-Ramirez A., Zhang J.Y. and Gardea-Torresdey J.L. (2012b), Stress response and tolerance of Zea mays to $\mathrm{CeO} 2$ nanoparticles: cross talk among $\mathrm{H}_{2} \mathrm{O}_{2}$, heat shock protein, and lipid peroxidation. ACS Nano, 6, 9615-9622.

Zhao L., Peralta-Videa J.R., Ren M., Varela-Ramirez A., Li C., Hernandez-Viezcas J.A., Aguilera R.J. and Gardea-Torresdey J.L. (2012a), Transport of $\mathrm{Zn}$ in a sandy loam soil treated with ZnO NPs and uptake by corn plants: electron microprobe and confocal microscopy studies, Chemical Engineering Journal, 184, 1-8.

Zimmermann P. and Zentgraf U. (2005), The correlation between oxidative stress and leaf senescence during plant development, Cellular and Molecular Biology Letters, 10, 515-534. 